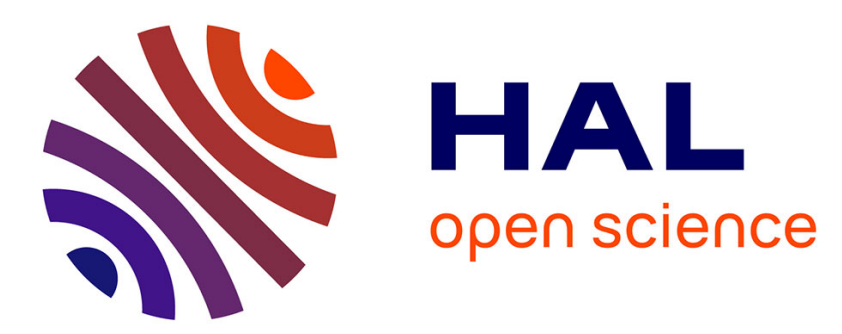

\title{
Kinematic and dynamic model based control of wheeled mobile manipulators: a unified framework for reactive approaches
}

\author{
Vincent Padois, Jean-Yves Fourquet, Pascale Chiron
}

\section{- To cite this version:}

Vincent Padois, Jean-Yves Fourquet, Pascale Chiron. Kinematic and dynamic model based control of wheeled mobile manipulators: a unified framework for reactive approaches. Robotica, 2007, 25 (2), pp.157-173. 10.1017/S0263574707003360 . hal-00578090

\section{HAL Id: hal-00578090 https://hal.science/hal-00578090}

Submitted on 18 Mar 2011

HAL is a multi-disciplinary open access archive for the deposit and dissemination of scientific research documents, whether they are published or not. The documents may come from teaching and research institutions in France or abroad, or from public or private research centers.
L'archive ouverte pluridisciplinaire HAL, est destinée au dépôt et à la diffusion de documents scientifiques de niveau recherche, publiés ou non, émanant des établissements d'enseignement et de recherche français ou étrangers, des laboratoires publics ou privés. 


\title{
Kinematic and dynamic model-based control of wheeled mobile manipulators: a unified framework for reactive approaches
}

\author{
V. PAdois ${ }^{\star}$, J.-Y. FourqueT ${ }^{\star \star}$, P. Chiron ${ }^{\star \star}$ \\ ${ }^{\star}$ Stanford Artificial Intelligence Laboratory \\ Stanford University, CA 94305, USA \\ vpadois@stanford.edu \\ ^^Laboratoire Génie de Production \\ Ecole Nationale d'Ingénieurs de Tarbes \\ 65000 Tarbes, France \\ (fourquet, pchiron)@enit.fr
}

\begin{abstract}
The work presented in this paper aims at providing a unified modeling framework for the reactive control of wheeled mobile manipulators (WMM). Where most work in the literature often provides models, sometimes simplified, of a given type of WMM, an extensive description of the obtaining of explicit kinematic and dynamic models of those systems is given. This modeling framework is particularly well suited for reactive control approaches which, in the case of mobile manipulation missions, are often necessary to handle the complexity of the tasks to be fulfilled, the dynamic aspect of the extended workspace and the uncertainties on the knowledge of the environment. A flexible reactive framework is thus also provided, allowing the sequencing of operational tasks (in our case tasks described in the end-effector frame) whose natures are different but also an on-line switching mechanism between constraints that are to be satisfied using the system redundancy. This framework has been successfully implemented in simulation and on a real robot. Some of the obtained results are presented.
\end{abstract}

\section{Introduction}

From manipulators executing highly specific and simple tasks in structured environments, robotics missions have evolved towards the service domain where robots are expected to explore (partially) unknown dynamic environments, 
interact with human beings or manipulate hazardous products. Examples of field or service robotics applications are numerous and they all involve robots whose workspace capabilities have to be extended and whose control architecture and strategies must ensure good overall performances in complex missions. The complexity of those missions lies in the fact that an extension of the workspace of robots leads to a partial knowledge of the environment: a precise map of a large environment cannot be known in advance and has to be updated at each time with the information regarding the dynamic elements of the environment (moving obstacles for example). It also lies in the fact that a complex mission is often an aggregation or sequence of different types of tasks: trajectory tracking, motion in dynamic environment, contact interaction, compliant motion. To fulfil such missions, robotic systems are required to combine both locomotion and manipulation capabilities and such systems are called mobile manipulators. The physical aspect of mobile manipulators varies with the type of mobile manipulation mission that is considered. The main differences between those systems of a same family consist in the locomotion mean that is used. Apart from very specific applications such as underwater applications where submarine locomotion is used, mobile manipulators can be divided into two large families: legged systems such as humanoid robots and wheeled systems. The need for humanoid robots is mostly associated to service missions for which the social aspect of the interaction is of great importance. To this social aspect of the interaction, one must add the need for adaptation to environments primarily designed for human beings. When those two characteristics are not present in the features of the mission to accomplish, the locomotion problem can be partially simplified by the use of wheeled mobile platforms whose design and control is less demanding. Moreover, in several cases, wheeled mobile platforms are more suitable than legged type robots whose locomotion properties are restricted especially in terms of speed and load. This paper focuses on this latter type of mobile manipulators: wheeled mobile manipulators (WMM), especially those evolving on flat grounds, i.e. whose platforms are not characterised by high terrain clearance capabilities. Any system combining a wheeled mobile platform and one or several manipulators (classically arms) is named WMM.

Those systems have the following features:

- wheeled mobile platforms, and by extension WMM, are nonholonomic systems;

- they are often kinematically redundant with respect to the task to be achieved;

- the mass properties, and thus the dynamic properties, of the wheeled platform and those of the manipulator(s) are very different.

To these characteristics, one has to add the constraints associated to the control of robots and robotic missions themselves:

- joint limits avoidance;

- rated input for the actuators; 
- singular configurations avoidance;

- obstacles avoidance;

- tip-over of the system and skidding / slipping of the wheels.

From this set of constraints and characteristics, different approaches have been developed to control WMM. A first class of approach is inherited from the control schemes that have been developed for manipulators. Those control schemes have been extended to WMM in order to account for their specificities. Among those approaches, the pioneering work of H. SERAJI ${ }^{1}$ can be distinguished. He proposed an extension of kinematic based control laws to the case of a mobile manipulator equipped with a wheeled platform (unicycle) and a manipulator. The innovation of this work was to consider the system without making any explicit difference between the two subsystems and thus to offer an implicit coordination control scheme, extending the concept of redundancy to the whole system and not only the manipulator. If for a given problem, one might want to use an ad hoc coordinated control scheme of a mobile manipulator (i.e. a control scheme where the coordination between the subsystems is suited to solve the given problem but may not be in other cases), the most generic framework to control mobile manipulators is clearly to consider an implicit coordination of the system. This framework allows to define the control problem without explicitly defining the wheeled platform trajectory thus not restricting the set of solutions to a smaller subset corresponding to a specific platform trajectory. This implicit coordination approach was extended to dynamic model based control schemes by Y. Yамамото and X. YUN in ${ }^{2,3}$ where they showed that the dynamic coupling between the two subsystems does not require to be fully compensated to achieve good tracking performances. Implicitly coordinated approaches to the force control problem of WMM has also been tackled using the dynamic models of some specific type of $\mathrm{WMM}^{4,5,6,7}$. Finally, reactive motion has been studied for WMM in the case of omnidirectional wheeled platforms using a dynamical model ${ }^{8}$. H. TANNER et al. also provided in ${ }^{9}$ some results for navigation with obstacles but from the planning point of view and considering the coordination of multiple WMM. If those studies provide some major developments for the control of WMM, they do not offer a general framework for the modelling of those systems. A unification of the kinematic modelling of those system is proposed by B. BAYLE et al. in ${ }^{10}$. This work is based on the generic classification of wheeled mobile platforms presented in ${ }^{11}$ by G. CAMPION et al.. M. FRUCHARD et al. also provide in ${ }^{12}$ a general kinematic control approach based on the transverse function approach that was initially developed for wheeled mobile robots ${ }^{13}$. This approach takes into account the small time controllability properties of wheeled mobile platforms and thus requires an explicit definition of the platform trajectory. In this paper, another solution is presented to solve this control problem. It is based on the instantaneous kinematics properties of the system. The goal is to provide a global model that relates the system's inputs to the motion and force at the end-effector. This does not require an explicit definition of the platform trajectory.

Regarding a generic dynamic model for WMM, no previous work has been done to propose a unified expression of 
this model. Of course, most of the studies of WMM based on a dynamic model provide an expression of this model in the specific case of a given robot. The goal of this paper is thus to provide an explicit formulation of the dynamic model of any kind of WMM that can be used for torque based control for reactive mission execution. An emphasis on the reactivity is put since it is, given the set of constraints to satisfy at any time, the complexity of the missions and the uncertainty on the plan execution, a necessary feature of the control of WMM.

This paper is organised in four sections. In the first section the basics of the generic configuration description of WMM is recalled. In the second section, after presenting the generic kinematic modelling of those systems that was proposed in ${ }^{14}$, a generic dynamic formulation of WMM and its connections to reactive control approaches is derived. In the third section, some results regarding reactive control of WMM in the case of robotic missions based on tasks sequencing are presented. This study is concluded by the perspectives and needs for future work regarding the reactive control of WMM.

\section{Configuration description of wheeled mobile manipulators}

Model based control approaches require a clear definition of the configuration parameters of the system whose models are derived. The configuration description of a mechanical system is a well known concept. However, even if the literature (see ${ }^{15}$ for example) in this domain is well furnished, it is of interest to take time to clarify this "simple" notion in order to provide a sound basis to any further developments. To do so, the general case of the configuration description of nonholonomic systems is treated and then focus is put on the case of WMM.

\subsection{General case of nonholonomic systems}

The configuration of a mechanical system is supposed to be known when the location (position and orientation) of any body of the system is known. In the $3 \mathrm{D}$ space, for a system composed of $n_{b}$ bodies, this description requires $6 n_{b}$ parameters $p_{1}, p_{2}, \ldots, p_{6 n_{b}}$ called primary generalized coordinates of the system. This set of coordinates is not unique and those parameters are usually not independent since constrained by the different joints between the bodies of the system or the bodies of the system and external bodies. Joints equations can be written in a generic form linking the primary generalized coordinates, their time derivatives and the time:

$$
\sum_{i=1}^{6 n_{b}} \alpha_{i}\left(p_{1}, p_{2}, \ldots, p_{6 n_{b}}, t\right) \dot{p}_{i}+\beta\left(p_{1}, p_{2}, \ldots, p_{6 n_{b}}, t\right)=0
$$

When equation (1) is integrable, it is called an holonomic joint equation and can be written:

$$
f\left(p_{1}, p_{2}, \ldots, p_{6 n_{b}}, t\right)=0
$$


If non integrable, (1) is called a nonholonomic joint equation. A mechanical system with one or more non integrable equation is called, by extension, a nonholonomic system.

Holonomic equations can be used to reduce the number of generalized coordinates required to describe the configuration of a system. Those equation are then called primary joints equations. Noting $h_{p}$ the number of primary joints equations chosen to reduce the number of parameters needed to describe the configuration of the system, this configuration is then defined by $n=6 n_{b}-h_{p}$ parameters that are the components of vector $\mathbf{q}$ called the vector of generalized coordinates. The number of nonholonomic joints equations is denoted $\bar{h}$. Those equations do not allow to reduce the size of $\mathbf{q}$ and together with the holonomic joints equations that have not be chosen to reduce the number of parameters needed to describe the configuration (whose number is noted $h_{c}$ ) of the system, they form the set of complementary joints equations. The global degree of freedom of the system is then given by $n_{d o f}=n-\left(h_{c}+\bar{h}\right)$ and only $n_{\text {dof }}$ components of vector q can have an independent evolution. The evolution of the $h_{c}+\bar{h}$ other ones is related to the latter by the set of complementary joints equations. Thus $\dot{\mathbf{q}}$, the vector of generalized velocities, is restricted to evolve in a subspace, whose size is $n_{d o f}$, tangent in $\mathbf{q}$ to the space associated to the generalized coordinates.

\subsection{Configuration of a wheeled mobile manipulator}

Considering the four classical types of wheels (fixed, centred, off-centred, Swedish) and using the notations introduced in ${ }^{11}$ (cf. figure 1), one can intuitively define the configuration of a wheeled mobile manipulator evolving on a planar surface as the configuration of the holonomic arm mounted on the mobile platform, the location of the wheeled mobile platform in the world reference frame and the configuration of each wheel with respect to the platform. For the sake of conciseness, the focus is put on the one arm case but extending the following models to the multiple arms case (i.e. multiple operational points case) is straightforward. This leads to:

$$
\mathbf{q}=\left[\begin{array}{llll}
\mathbf{q}_{m}^{t} & \boldsymbol{\xi}_{p}^{t} & \boldsymbol{\varphi}^{t} & \boldsymbol{\beta}^{t}
\end{array}\right]^{t}
$$

where:

$-\mathbf{q}_{m}$ is the configuration of the manipulator mounted on the platform. Using the modified version of the D.-H. parameters ${ }^{16}$, the $n_{m}$ components of $\mathbf{q}_{m}$ can be naturally chosen as the $n_{m}$ joint parameters thus leading to a minimal representation of the configuration of the manipulator;

$-\boldsymbol{\xi}_{p}=\left[\begin{array}{lll}x & y & \vartheta\end{array}\right]^{t}$ is the vector of location of the platform (whose size is denoted $m_{p}=3$ ) with respect to the world reference frame $\mathcal{R}=(O, \vec{x}, \vec{y}, \vec{z})$;

$-\varphi=\left[\begin{array}{llll}\varphi_{f}^{t} & \varphi_{c}^{t} & \varphi_{o c}^{t} & \varphi_{s}^{t}\end{array}\right]^{t}$ is the vector of the $N=N_{f}+N_{c}+N_{o c}+N_{s}$ spinning angles of the $N_{f}$ fixed wheels, $N_{c}$ centred wheels, $N_{o c}$ off-centred wheels and $N_{s}$ Swedish wheels respectively; 


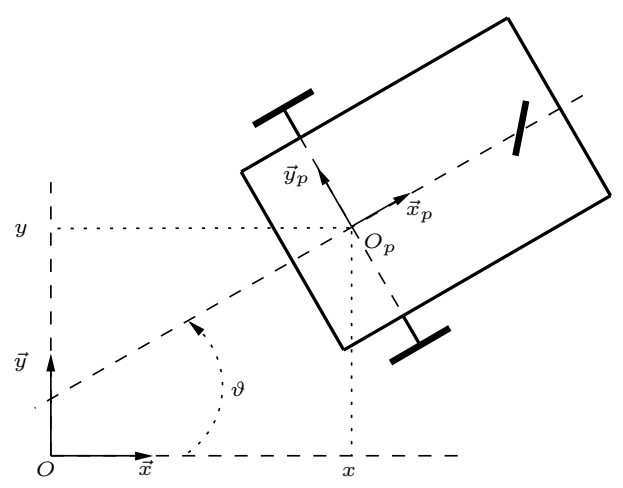

a) location of a planar platform

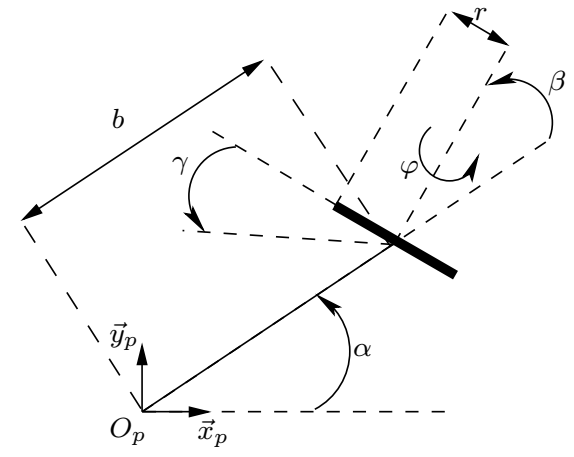

b) parameters for fixed, centred and Swedish wheels description

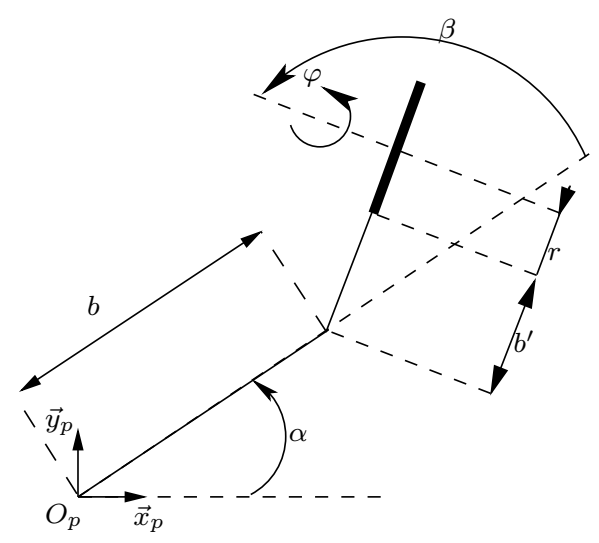

c) parameters for off-centred wheel description

Figure 1: Configuration description of wheeled planar platforms.

$-\boldsymbol{\beta}=\left[\begin{array}{ll}\boldsymbol{\beta}_{o c}^{t} & \boldsymbol{\beta}_{c}^{t}\end{array}\right]^{t}$ is the vector of the $N_{S}=N_{c}+N_{o c}$ steering angles of the $N_{c}$ centred wheels and $N_{o c}$ off-centred wheels respectively.

The configuration of a WMM is thus defined by a vector $\mathbf{q}$ of size $n=n_{m}+m_{p}+N+N_{S}$. However, apart from classical revolute and prismatic joints, a WMM is subject to rolling without slipping conditions which are joints equations of a specific type. Those conditions are hereafter described.

\subsection{Rolling without slipping conditions}

The rolling without slipping conditions of a wheel represent the fact that perfect joint of the wheel with the ground is considered, i.e. no slipping of the wheel in its vertical plane and no skidding in its orthogonal plane. Using the configuration description, they can respectively be written, for a given wheel:

$$
\left[\begin{array}{ccc}
-S_{\alpha \beta \gamma} & C_{\alpha \beta \gamma} & b C_{\beta \gamma}
\end{array}\right] R(\vartheta)^{T} \dot{\boldsymbol{\xi}}_{p}+r C_{\gamma} \dot{\varphi}=0
$$




$$
D\left(\mathbf{q}_{p}\right)=\left[\begin{array}{ccccc}
\mathcal{O}_{m \times m} & \mathcal{O}_{m \times m_{p}} & \mathcal{O}_{m \times N} & \mathcal{O}_{m \times N_{o c}} & \mathcal{O}_{m \times N_{c}} \\
\mathcal{O}_{N \times m} & J_{1} R(\vartheta)^{T} & J_{2} & \mathcal{O}_{N \times N_{o c}} & \mathcal{O}_{N \times N_{c}} \\
\mathcal{O}_{N_{f} \times m} & C_{1 f} & \mathcal{O}_{N_{f} \times N} & \mathcal{O}_{N_{f} \times N_{o c}} & \mathcal{O}_{N_{f} \times N_{c}} \\
\mathcal{O}_{N_{c} \times m} & C_{1 c}\left(\boldsymbol{\beta}_{c}\right) R(\vartheta)^{T} & \mathcal{O}_{N_{c} \times N} & \mathcal{O}_{N_{c} \times N_{o c}} & \mathcal{O}_{N_{c} \times N_{c}} \\
\mathcal{O}_{N_{o c} \times m} & C_{1 o c}\left(\boldsymbol{\beta}_{o c}\right) R(\vartheta)^{T} & \mathcal{O}_{N_{o c} \times N} & C_{2 o c} & \mathcal{O}_{N_{o c} \times N_{c}}
\end{array}\right]
$$

Table 1: Detailed description of matrix $D\left(\mathbf{q}_{p}\right)$.

and:

$$
\left[\begin{array}{ccc}
C_{\alpha \beta} & S_{\alpha \beta} & b^{\prime}+b S_{\beta}
\end{array}\right] R(\vartheta)^{T} \dot{\boldsymbol{\xi}}_{p}+b^{\prime} \dot{\beta}=0,
$$

where (4) does not apply to Swedish wheels, $C_{\alpha \beta}$ and $S_{\alpha \beta}$ are short notations respectively for $\cos (\alpha+\beta)$ and $\sin (\alpha+\beta) . R(\vartheta)$ represents the rotation matrix of angle $\vartheta$ around $\vec{z}$.

Those joints equations are most of the time non integrable, i.e. nonholonomic. In the specific cases where some combinations of those equations can be integrated, they are often kept as complementary joints equations. This choice can be justified in the practical cases where pure rolling without slipping is never completely respected and thus basing the knowledge of some configuration parameters on this assumption might lead to a drift of the reconstructed (not directly measured by physical sensors) parameters.

\section{Unified kinematic and dynamic modelling of wheeled mobile manip- ulators}

Given a WMM, the task of the system is expressed in terms of a trajectory to follow, a point to reach or a force to exert for the end-effector of the manipulator. This operational point is associated to a set of operational coordinates whose size is associated to the type of task to fulfil: constrained location, constrained position etc. The vector of operational coordinates of the manipulator is written $\boldsymbol{\xi}$ and its size is denoted $m$. The forward kinematics model of the WMM can then be written:

$$
\boldsymbol{\xi}=\mathbf{f}\left(\boldsymbol{\xi}_{p}, \mathbf{q}_{m}\right)
$$

where $\mathbf{f}$ is a set of nonlinear functions, independently of the type of platform (cf. figure 2).

Differentiation of (5) leads to:

$$
\dot{\xi}=J(\mathbf{q}) \dot{\mathbf{q}}
$$

$J(\mathbf{q})$ is the $m \times n$ Jacobian matrix of the WMM.

Equation (6) is not sufficient to fully describe the system's differential kinematics. One needs to also take the rolling 


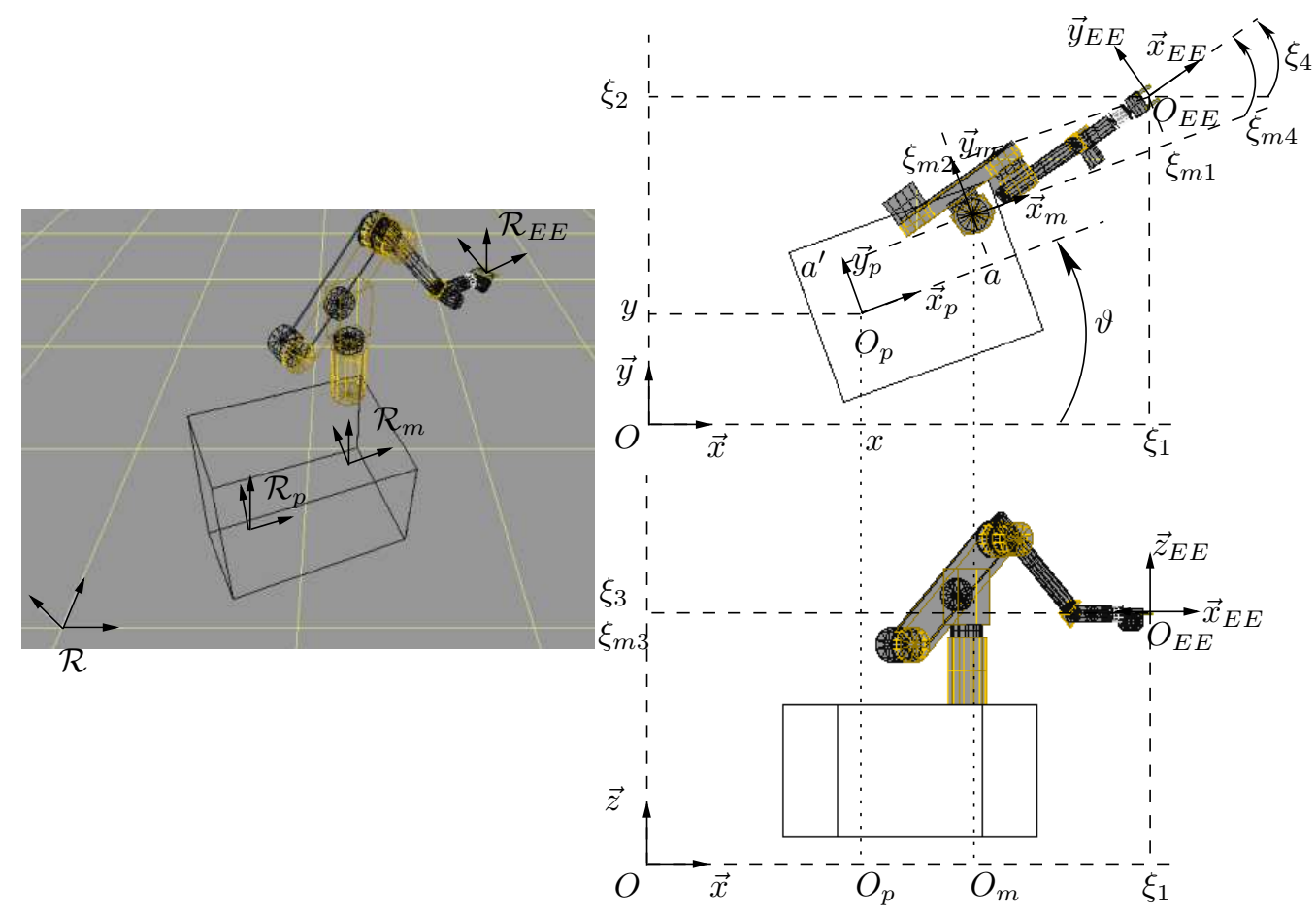

Figure 2: Parameters of a WMM defining the forward kinematics model.

without slipping joint equation of the wheels. Those equations can be written in the concise form:

$$
\begin{gathered}
{\left[\begin{array}{c}
J_{1 f} \\
J_{1 c}\left(\boldsymbol{\beta}_{c}\right) \\
J_{1 o c}\left(\boldsymbol{\beta}_{o c}\right) \\
J_{1 s}
\end{array}\right] R(\vartheta)^{T} \dot{\boldsymbol{\xi}}_{p}+\left[\begin{array}{c}
J_{2 f} \\
J_{2 c} \\
J_{2 o c} \\
J_{2 s}
\end{array}\right] \dot{\boldsymbol{\varphi}}=0,} \\
{\left[\begin{array}{c}
C_{1 f} \\
C_{1 c}\left(\boldsymbol{\beta}_{c}\right) \\
C_{1 o c}\left(\boldsymbol{\beta}_{o c}\right)
\end{array}\right] R(\vartheta)^{T} \dot{\boldsymbol{\xi}}_{p}+\left[\begin{array}{c}
\mathcal{O}_{N_{f} \times N_{o c}} \\
\mathcal{O}_{N_{c} \times N_{o c}} \\
C_{2 o c}
\end{array}\right] \dot{\boldsymbol{\beta}}_{o c}=0 .}
\end{gathered}
$$

One can easily deduce that :

$-J_{1}=\left[\begin{array}{llll}J_{1 f}^{T} & J_{1 c}\left(\boldsymbol{\beta}_{c}\right)^{T} & J_{1 o c}\left(\boldsymbol{\beta}_{o c}\right)^{T} & J_{1 s}^{T}\end{array}\right]^{T}$ is a $\left(N \times m_{p}\right)$ matrix;

$-J_{2}=\left[\begin{array}{llll}J_{2 f}^{T} & J_{2 c}^{T} & J_{2 o c}^{T} & J_{2 s}^{T}\end{array}\right]^{T}$ is a diagonal, constant, $(N \times N)$ matrix;

$-C_{1}=\left[\begin{array}{lll}C_{1 f}^{T} & C_{1 c}\left(\boldsymbol{\beta}_{c}\right)^{T} & C_{1 o c}\left(\boldsymbol{\beta}_{o c}\right)^{T}\end{array}\right]^{T}$ is a $\left(\left(N-N_{s}\right) \times m_{p}\right)$ matrix

- and $C_{2}=\left[\begin{array}{lll}\mathcal{O}_{N_{o c} \times N_{f}} & \mathcal{O}_{N_{o c} \times N_{c}} & C_{2 o c}^{T}\end{array}\right]^{T}$ is a diagonal, constant, $\left(\left(N-N_{s}\right) \times N_{o c}\right)$ matrix;

(7) and (8) can then be compactly written as: 


$$
D\left(\mathbf{q}_{p}\right) \dot{\mathbf{q}}=0,
$$

where a full description of $D\left(\mathbf{q}_{p}\right)$ is given in table 1.

In order to be able to control the system without violating the rolling without slipping assumption, it is necessary to find the subset of $\dot{\mathbf{q}}$ such that (9) is always satisfied.

\subsection{Mobility analysis}

An analysis of matrix $D\left(\mathbf{q}_{p}\right)$ leads to the conclusion that for a motion of a WMM to be possible, conditions to be respected are only related to the velocity of the wheeled platform. Especially, for a possible motion of the platform to exist, the following constraint, extracted from $D\left(\mathbf{q}_{p}\right)$, has to be met:

$$
C_{1 f c}\left(\boldsymbol{\beta}_{c}\right) R(\vartheta)^{T} \dot{\boldsymbol{\xi}}_{p}=0
$$

where :

$$
C_{1 f c}\left(\boldsymbol{\beta}_{c}\right)=\left[\begin{array}{c}
C_{1 f} \\
C_{1 c}\left(\boldsymbol{\beta}_{c}\right)
\end{array}\right] .
$$

This condition on the movement of the platform is such that $\dot{\boldsymbol{\xi}}_{p}$ is constrained to evolve in a subspace of the space of possible planar velocities. The size of this subspace is denoted $\delta_{m o b p} \leq m_{p}$ and called the degree of mobility of the platform. $\delta_{\text {mobp }}$ is dependent on the platform type and it is shown in ${ }^{11}$ that there is actually a limited number of wheeled platform types such that $\delta_{m o b p} \neq 0$, i.e. such that the platform can move. Considering the set of feasible wheeled platform type described in ${ }^{11}$ (i.e. wheeled platform such that $\operatorname{rank}\left(C_{1 f c}\left(\boldsymbol{\beta}_{c}\right)\right)<m_{p}$ ), constraint (10) requires to choose $\dot{\boldsymbol{\xi}}_{p}$ in the range of $\mathcal{N}\left(C_{1 f c}\left(\boldsymbol{\beta}_{c}\right)\right)$. Given, $\Sigma\left(\boldsymbol{\beta}_{c}\right)$ a $\left(m_{p} \times \delta_{\text {mobp }}\right)$ matrix whose column are a basis of $\mathcal{N}\left(C_{1 f c}\left(\boldsymbol{\beta}_{c}\right)\right)$, any $\dot{\boldsymbol{\xi}}_{p}$ chosen as :

$$
\dot{\boldsymbol{\xi}}_{p}=R(\vartheta) \Sigma\left(\boldsymbol{\beta}_{c}\right) \mathbf{u}_{m o b p}
$$

will satisfy constraint (10). $\mathbf{u}_{\text {mobp }}$ is any vector of size $\delta_{\text {mobp }}$ and is named the mobility control input of the wheeled platform. From relation (11), the values of the velocity $\dot{\boldsymbol{\varphi}}$ and $\dot{\boldsymbol{\beta}}_{o c}$ that will lead to the complete satisfaction of constraint (9) are then obtained by :

$$
\dot{\boldsymbol{\varphi}}=-J_{2}^{-1} J_{1} \Sigma\left(\boldsymbol{\beta}_{c}\right) \mathbf{u}_{m o b p},
$$

and :

$$
\dot{\boldsymbol{\beta}}_{o c}=-C_{2 o c}^{-1} C_{1 o c} \Sigma\left(\boldsymbol{\beta}_{c}\right) \mathbf{u}_{m o b p} .
$$


Constraint (9) does not give any guidance for the determination of $\dot{\boldsymbol{\beta}}_{c}$, but among the set of feasible wheeled platform type, one can demonstrate that the number of components of $\dot{\boldsymbol{\beta}}_{c}$ that can be steered independently cannot be more that 2. This number is called the degree of steerability of the wheeled platform and is denoted $\delta_{s t}$. The $\delta_{s t}$ independent components of $\dot{\boldsymbol{\beta}}_{c}$ form the steerability control input $\mathbf{u}_{s t}$ of the wheeled platform. The steering velocity of any other centred wheel can be determined from this steerability control vector (see ${ }^{17}$ ).

Regarding the manipulator, its mobility control input can be simply chosen as :

$$
\mathbf{u}_{m o b m}=\dot{\mathbf{q}}_{m}
$$

The sum of $\delta_{m o b}+\delta_{s t}$ is called the degree of manoeuvrability $\delta_{\text {man }}$ of the WMM. Given the restriction on the type of feasible wheeled platform and the definition of the degree of freedom of a mechanical system recalled in 2.1 :

$$
\delta_{\text {man }}=n_{\text {dof }} .
$$

Considering wheeled platform with no more than two steerable wheels (any other case can be deduced from this one), the subset of $\dot{\mathbf{q}}$ satisfying (9) are thus related to the control input of the WMM by the relation :

$$
\dot{\mathbf{q}}=S\left(\mathbf{q}_{p}\right) \mathbf{u},
$$

where $\mathbf{u}=\left[\begin{array}{lll}\mathbf{u}_{m o b m}^{t} & \mathbf{u}_{m o b p}^{t} & \mathbf{u}_{s t}^{t}\end{array}\right]^{t}$ and :

$$
S\left(\mathbf{q}_{p}\right)=\left[\begin{array}{ccc}
\mathcal{I}_{m} & \mathcal{O}_{m \times \delta_{m o b p}} & \mathcal{O}_{m \times \delta_{s t}} \\
\mathcal{O}_{m_{p} \times m} & R(\vartheta) \Sigma\left(\boldsymbol{\beta}_{c}\right) & \mathcal{O}_{m_{p} \times \delta_{s t}} \\
\mathcal{O}_{N \times m} & -J_{2}^{-1} J_{1} \Sigma\left(\boldsymbol{\beta}_{c}\right) & \mathcal{O}_{N \times \delta_{s t}} \\
\mathcal{O}_{N_{o c} \times m} & -C_{2 o c}^{-1} C_{1 o c} \Sigma\left(\boldsymbol{\beta}_{c}\right) & \mathcal{O}_{N_{o c} \times \delta_{s t}} \\
\mathcal{O}_{\delta_{s t} \times m} & \mathcal{O}_{\delta_{s t} \times \delta_{m o b p}} & \mathcal{I}_{\delta_{s t}}
\end{array}\right]
$$

One can easily check that $D\left(\mathbf{q}_{p}\right) S\left(\mathbf{q}_{p}\right)=0$ for any $\mathbf{q}$. An analysis of the number of joints equations that are actually nonholonomic can be determined by studying the span of the vector fields generated by the columns of $S\left(\mathbf{q}_{p}\right)$ (details can be found in ${ }^{11}$ and examples in the case of WMM in ${ }^{18}$ ).

\subsection{Kinematic model}

From this subset of generalized velocities, the kinematic model can now be written in a form that can be used to control the system. It is first to be noted that there is no kinematic connection between $\dot{\boldsymbol{\xi}}$ and $\dot{\boldsymbol{\beta}}_{c}$. Thus, even if the problem of designing the proper steerability control input for the WMM is not tackled in this paper, it is of 
importance to notice that the full kinematic model description of a WMM is extended to the following relation:

$$
\dot{\mathbf{z}}=B(\mathbf{q}) \dot{\mathbf{q}}
$$

where :

$$
\mathbf{z}=\left[\begin{array}{c}
\boldsymbol{\xi} \\
\boldsymbol{\beta}_{c}
\end{array}\right]
$$

and :

$$
B(\mathbf{q})=\left[\begin{array}{cc}
J(\mathbf{q}) & \\
\mathcal{O}_{\delta_{s t} \times\left(n-\delta_{s t}\right)} & \mathcal{I}_{\delta_{s t}}
\end{array}\right]
$$

Plugging (15) in (16) leads to :

$$
\dot{\mathbf{z}}=\bar{B}(\mathbf{q}) \mathbf{u}
$$

where :

$$
\bar{B}(\mathbf{q})=B(\mathbf{q}) S\left(\mathbf{q}_{p}\right)
$$

which can also be written :

$$
\bar{B}(\mathbf{q})=\left[\begin{array}{cc}
\bar{J}(\mathbf{q}) & \mathcal{O}_{m \times \delta_{s t}} \\
\mathcal{O}_{\delta_{s t} \times \delta_{m o b}} & \mathcal{I}_{\delta_{s t}}
\end{array}\right],
$$

with :

$$
\left[\begin{array}{ll}
\bar{J}(\mathbf{q}) & \mathcal{O}_{m \times \delta_{s t}}
\end{array}\right]=J(\mathbf{q}) S\left(\mathbf{q}_{p}\right) .
$$

In the kinematic redundant case $\left(\right.$ i.e. $\delta_{m o b}>m$ ), an inverse kinematic model-based controller can be used :

$$
\mathbf{u}=\bar{B}(\mathbf{q})^{\star} \dot{\mathbf{z}}+\left(\mathcal{I}_{n_{d o f}}-\bar{B}(\mathbf{q})^{\star} \bar{B}(\mathbf{q})\right) \mathbf{u}_{0},
$$

with $\bar{B}(\mathbf{q})^{\star}$ a weighted pseudo-inverse of $\bar{B}(\mathbf{q})$, $\dot{\mathbf{z}}$ the desired operational and steering velocity and $\mathbf{u}_{0}$ any vector. $\bar{B}(\mathbf{q})^{\star}$ is given by :

$$
\bar{B}(\mathbf{q})^{\star}=\left[\begin{array}{cc}
\bar{J}(\mathbf{q})^{\star} & \mathcal{O}_{\delta_{m o b} \times \delta_{s t}} \\
\mathcal{O}_{\delta_{s t} \times m} & \mathcal{I}_{\delta_{s t}}
\end{array}\right] .
$$

The computed $\mathbf{u}$ has the minimum least-squares norm with respect to the chosen weight (see ${ }^{19}$ ). The choice of the weight is thus very important since it will determine the way the actuators are solicited. For example, choosing a basic pseudo-inverse leads to an equivalent solicitation of all the actuators without taking into account their respective sizes and positions in the kinematic chain. This is obviously not the best solution given the difference in size of an actuator used to spin one of the platform's wheels and an actuator used for the motion of the manipulator 
wrist. To take this kind of differences into account, the weighting matrix can be related to the way inertia is distributed in the system. More generally, different weighting matrices can be used to achieve different goals. An example of possible choice is given in the part of this paper dealing with dynamics (3.3).

Access to the system redundancy is given by the term $\left(\mathcal{I}_{n_{\text {dof }}}-\bar{B}(\mathbf{q})^{\star} \bar{B}(\mathbf{q})\right) \mathbf{u}_{0}$. $\mathbf{u}_{0}$ can be chosen using gradient descent techniques to locally minimize any potential function $\mathcal{P}(\mathbf{q})$ used to represent a constraint that has to be satisfied by the system at a given time. To locally minimize $\mathcal{P}(\mathbf{q}), \dot{\mathcal{P}}(\mathbf{q}) \leq 0$ has to be ensured. $\dot{\mathcal{P}}(\mathbf{q})$ can be expressed as :

$$
\dot{\mathcal{P}}(\mathbf{q})=\nabla^{t} \mathcal{P}(\mathbf{q}) \dot{\mathbf{q}}
$$

where $\boldsymbol{\nabla} \mathcal{P}(\mathbf{q})$ is the gradient of function $\mathcal{P}(\mathbf{q})$. In order not to provide any operational effects and to be compatible with the complementary joints equations, $\dot{\mathbf{q}}$ has to be chosen as :

$$
\dot{\mathbf{q}}=S\left(\mathbf{q}_{p}\right)\left(\mathcal{I}_{n_{d o f}}-\bar{B}(\mathbf{q})^{\star} \bar{B}(\mathbf{q})\right) \mathbf{u}_{0}
$$

This leads to :

$$
\dot{\mathcal{P}}(\mathbf{q})=\nabla^{t} \mathcal{P}(\mathbf{q}) S\left(\mathbf{q}_{p}\right)\left(\mathcal{I}_{n_{d o f}}-\bar{B}(\mathbf{q})^{\star} \bar{B}(\mathbf{q})\right) \mathbf{u}_{0}
$$

Given the idempotence property of matrix projectors such as $\left(\mathcal{I}_{n_{d o f}}-\bar{B}(\mathbf{q})^{\star} \bar{B}(\mathbf{q})\right)$, choosing $\mathbf{u}_{0}$ as :

$$
\mathbf{u}_{0}=-K\left(\nabla^{t} \mathcal{P}(\mathbf{q}) S\left(\mathbf{q}_{p}\right)\left(\mathcal{I}_{n_{d d l}}-\bar{B}(\mathbf{q})^{\star} \bar{B}(\mathbf{q})\right)\right)^{t}
$$

where $K$ is a positive definite matrix, leads to $\dot{\mathcal{P}}(\mathbf{q}) \leq 0$. One has to notice that when the operational task to be achieved and the potential function to be optimised are not compatible, the projection of $\mathbf{u}_{0}$ into $\left(\mathcal{I}_{n_{\text {dof }}}-\bar{B}(\mathbf{q})^{\star} \bar{B}(\mathbf{q})\right)$ will lead to 0 . The operational task has a greater priority than the optimisation of the potential function. The priority concept can be extended to multiple secondary tasks achieved (or partially achieved) at the same time. When the secondary tasks can be expressed as potential functions, a solution consists in building a weighted-sum of the different potential functions. The potential function with the biggest priority is given the biggest weight, the one with the minimum priority is given the smallest weight ${ }^{20}$. This kind of approach can be efficient for some specific problems but one should keep in mind that the optimised criterion has no clear signification and in the case of critical secondary tasks, these methods generally lead to failure. A better approach to solve this problem is based on recursive null space projections of the different tasks with respect to their levels of priority. The basics of this prioritization technique can be found in ${ }^{21}$ but this is still an open problem which is being addressed by some recent publications $:^{22},{ }^{23}$. These studies attempt to design algorithms to arbitrate the fulfillment of different secondary tasks (among which some can be partially or completely conflicting) with different priorities. Those techniques make a lot of sense with highly redundant robots such as humanoids. In the case of WMM, the redundancy degree of the 
system is not high enough to efficiently optimise multiple potential functions at the same time. Thus, in this paper, only one secondary task is considered at a time and switching occurs when the fulfillment of another secondary task becomes more critical. Of course, this approach does not solve the redundancy problem in a completely generic manner and can lead to some failure cases. However, it is generic enough to provide an efficient solution to a large number of missions.

\subsection{Dynamic model}

When interaction forces are involved and/or compliant behaviour is desired, the use of a dynamic model of the system is required in order to correctly compensate for the natural dynamic of the system. The implicit and explicit formulation of the dynamics of manipulators is a widely treated subject. Regarding WMM, some papers propose a dynamic modelling in the specific case of a given system (see for example ${ }^{2}$ or $^{6}$ ). In the case of a specific WMM, J.H. Chung et al. in ${ }^{24}$ extended, using a tire friction model, the dynamic description to the case where the rolling without slipping conditions are not always respected. Some work was also done to propose a general description of the dynamics of a WMM: H. TANNER et al. in ${ }^{25}$ proposed a formulation of the dynamic model of WMM using KANE's approach. A recursive formulation of the dynamics of a WMM was also proposed in Q. YU et al. in $^{26}$ with an application to a specific example of WMM. These studies are of high interest since they give a very methodological approach to derive a dynamic description of WMM, however, these formulations, if they are well suited to simulate the dynamics of such systems, cannot be directly used for control purposes. Such an explicit dynamic model based on LAGRANGE formulation is here derived. This only partially covers the topic of the dynamic modelling of nonholonomic systems and ${ }^{27}$ and ${ }^{28}$ can be used as complete reference books.

The LAGRANGE formulation of the dynamic of a mechanical system can be summarised by the following formula:

$$
\frac{d}{d t}\left(\frac{\partial T(\mathbf{q}, \dot{\mathbf{q}}, t)}{\partial \dot{q}_{i}}\right)-\left(\frac{\partial T(\mathbf{q}, \dot{\mathbf{q}}, t)}{\partial q_{i}}\right)=Q_{i}, \quad(1 \leq i \leq n),
$$

or :

$$
P_{i}=Q_{i}, \quad(1 \leq i \leq n)
$$

with $P_{i}=\frac{d}{d t}\left(\frac{\partial T(\mathbf{q}, \dot{\mathbf{q}}, t)}{\partial \dot{q}_{i}}\right)-\left(\frac{\partial T(\mathbf{q}, \dot{\mathbf{q}}, t)}{\partial q_{i}}\right)$

This results from the expression of the virtual power principle, expressed in terms of the kinetic energy $T(\mathbf{q}, \dot{\mathbf{q}}, t)$ of the system. Briefly :

- $P_{i}$ is the force applied to the system which is due to its motion and which is associated to coordinate $q_{i}$;

- $Q_{i}$ is the sum of any other force acting on the system and associated to coordinate $q_{i}$.

However relation (21) does not account for the complementary joints equations and any virtual velocity $\dot{\mathbf{q}}^{\star}$ compa- 
tible with the complementary joints equations is such that :

$$
D(\mathbf{q}) \dot{\mathbf{q}}^{\star}=0 .
$$

The virtual power principle also leads to the following results :

$$
(\mathbf{P}-\mathbf{Q})^{t} \dot{\mathbf{q}}^{\star}=0
$$

Relations (22) and (23) leads to the conclusion that $\dot{\mathbf{q}}^{\star}$ has to be orthogonal to the $\bar{h}+h_{c}$ rows of $D(\mathbf{q})$ and also orthogonal to vector $(P-Q)$. The latter can thus be written as a linear combination of $D(\mathbf{q})$ rows:

$$
P_{i}-Q_{i}=\sum_{j=1}^{\bar{h}+h_{c}} \lambda_{j} D_{j i},(1 \leq i \leq n),
$$

where $\lambda_{j}$ are $\bar{h}+h_{c}$ scalars (historically known as LAGRANGE multipliers).

Complete dynamics of the system is thus given by:

$$
P_{i}=Q_{i}+\sum_{j=1}^{\bar{h}+h_{c}} \lambda_{j} D_{j i}, \quad(1 \leq i \leq n)
$$

Computing the kinetic energy of the system and computing the components of $P$, dynamics of a WMM can always be written in the form:

$$
A(\mathbf{q}) \ddot{\mathbf{q}}+C(\mathbf{q}, \dot{\mathbf{q}}) \dot{\mathbf{q}}=\mathbf{Q}+D(\mathbf{q})^{t} \boldsymbol{\lambda}
$$

$A(\mathbf{q})$ is the $n \times n$ symmetric, positive definite, inertia matrix of the system. $C(\mathbf{q}, \dot{\mathbf{q}})$ is the $n \times n$ CoRIOLIS and centrifugal matrix of the system. $\mathbf{Q}$ can be written as the sum of four terms $\mathbf{Q}=\mathbf{Q}_{a}+\mathbf{Q}_{g}+\mathbf{Q}_{d}+\mathbf{Q}_{\text {ext }}$, where :

$-\mathbf{Q}_{a}=M \boldsymbol{\Gamma}$, the actuation forces acting on the system. An analysis of the actuation of WMM is not provided here (see ${ }^{11}$ ) but assuming a system with $n_{a}$ actuators such that all control inputs in $\mathbf{u}$ can be independently actuated, $\boldsymbol{\Gamma}$ is the actual vector of actuation torques (actually force or torque) and $M$ is a $\left(n \times n_{a}\right)$ matrix such that $M_{i k}=1$ if coordinate $q_{i}$ is directly actuated by actuator $k$ and $M_{i k}=0$ otherwise;

$-\mathbf{Q}_{g}=-\mathbf{g}(\mathbf{q})$, the vector of gravity forces acting on the system;

$-\mathbf{Q}_{d}$, the vector of force disturbances such as friction effects acting on the system;

$-\mathbf{Q}_{\text {ext }}$, the vector of external interaction forces. 
Regarding $\mathbf{Q}_{e x t}$, the location $\boldsymbol{\xi}_{c_{l}}$ of the interaction/contact on the WMM can be related to the configuration by a geometrical relation:

$$
\boldsymbol{\xi}_{c_{l}}=\mathbf{g}_{c_{l}}(\mathbf{q})
$$

that can be differentiated leading to a kinematic relation of the type:

$$
\dot{\boldsymbol{\xi}}_{c_{l}}=J_{c_{l}}(\mathbf{q}) \dot{\mathbf{q}}
$$

The kineto-static principle of equivalence allows to compute $\mathbf{Q}_{\text {ext }}$, the equivalent generalized force associated to this contact force at $\boldsymbol{\xi}_{c_{l}}$, as a function of the contact forces $\mathbf{f}_{c_{l}}$. This leads to:

$$
\mathbf{Q}_{e x t}=-J_{c_{l}}(\mathbf{q})^{t} \mathbf{f}_{c_{l}}
$$

For multiple contact points, one can concatenate the vectors of contact forces $\mathbf{f}_{c_{l}}$ and jacobians $J_{c_{l}}(\mathbf{q})$ and thus for $n_{c}$ contact locations:

$$
\mathbf{Q}_{e x t}=-J_{c}(\mathbf{q})^{t} \mathbf{f}_{c}
$$

where:

$$
J_{c}(\mathbf{q})=\left(\begin{array}{llllll}
J_{c_{1}}(\mathbf{q}) & \ldots & J_{c_{l}}(\mathbf{q}) & \ldots & J_{c_{n_{c}}}(\mathbf{q})
\end{array}\right)^{t}
$$

and:

$$
\mathbf{f}_{c}=\left(\begin{array}{lllll}
\mathbf{f}_{c_{1}} & \ldots & \mathbf{f}_{c_{l}} & \ldots & \mathbf{f}_{c_{n_{c}}}
\end{array}\right)^{t}
$$

No assumption about the compatibility between the contact force applied at $\boldsymbol{\xi}_{c}$ and the motion of the controlled system is made here. For a detailed multi-contact force control study, see ${ }^{29}$. The focus is rather put here on the specific case where this contact interaction occurs at the end-effector of the WMM but also assume some resistive torque for the steering axes of the steerable wheels. This latter assumption is realistic when the contact point of the wheels with the ground is actually more a line than a point thus leading to some amplified resistive friction effects of the ground on the wheels with respect to the steering axis. In that specific case, $J_{c}(\mathbf{q})=B(\mathbf{q})$ and thus:

$$
\mathbf{Q}_{e x t}=-B(\mathbf{q})^{t} \mathbf{f}_{c}
$$

From here, neglecting friction, the dynamic equation of motion can be written:

$$
A(\mathbf{q}) \ddot{\mathbf{q}}+C(\mathbf{q}, \dot{\mathbf{q}}) \dot{\mathbf{q}}+\mathbf{g}(\mathbf{q})+B(\mathbf{q})^{t} \mathbf{f}_{c}=M \boldsymbol{\Gamma}+D(\mathbf{q})^{t} \boldsymbol{\lambda}
$$

$D(\mathbf{q})^{t} \boldsymbol{\lambda}$ is actually the projection of the reaction forces at each joint whose equation has been chosen as a com- 
plementary joint equation. Assuming that only the rolling without slipping joint equation have been kept as complementary joints equations, $\boldsymbol{\lambda}$ precisely represents the vector of the tangential reaction forces (collinear with plane $(O, \vec{x}, \vec{y}))$ of the wheels on the ground. If the non slipping assumption is not realistic (that can be the case in outdoor, non-structured ground applications), using a friction model, one can lead a study of the slipping/skidding conditions for the system based on (25). In some applications, this assumption is valid and one may want to restrict the study to those motions that do not violate the constraints. To do so, one can eliminate the term $D(\mathbf{q})^{t} \boldsymbol{\lambda}$ in (25) by multiplying both sides by $S(\mathbf{q})^{t}$. This leads to:

$$
\begin{gathered}
S(\mathbf{q})^{t} A(\mathbf{q}) \ddot{\mathbf{q}}+S(\mathbf{q})^{t} C(\mathbf{q}, \dot{\mathbf{q}}) \dot{\mathbf{q}}+ \\
S(\mathbf{q})^{t} \mathbf{g}(\mathbf{q})+S(\mathbf{q})^{t} B(\mathbf{q})^{t} \mathbf{f}_{c}=S(\mathbf{q})^{t} M \boldsymbol{\Gamma}
\end{gathered}
$$

The rolling without slipping conditions are supposed to be respected and thus $\dot{\mathbf{q}}$ and $\ddot{\mathbf{q}}$ are restricted to:

$$
\dot{\mathbf{q}}=S(\mathbf{q}) \mathbf{u} \text { and } \ddot{\mathbf{q}}=\dot{S}(\mathbf{q}) \mathbf{u}+S(\mathbf{q}) \dot{\mathbf{u}}
$$

Plugging into (26) leads to the reduced dynamic model of the WMM or constraint consistent dynamic model:

$$
\bar{A}(\mathbf{q}) \dot{\mathbf{u}}+\bar{C}(\mathbf{q}, \dot{\mathbf{q}}) \mathbf{u}+\overline{\mathbf{g}}(\mathbf{q})+\bar{B}(\mathbf{q})^{t} \mathbf{f}_{c}=\overline{\boldsymbol{\Gamma}}
$$

where:

$-\bar{A}(\mathbf{q})$ is the $n_{\text {dof }} \times n_{\text {dof }}$, symmetric, positive definite, constraint consistent inertia matrix of the system. It can be decomposed as:

$$
\bar{A}(\mathbf{q})=\left[\begin{array}{cc}
A_{m}\left(\mathbf{q}_{m}\right) & \bar{A}_{p / m}(\mathbf{q}) \\
\bar{A}_{p / m}(\mathbf{q})^{t} & \bar{A}_{p}(\mathbf{q})+\bar{A}_{m p}(\mathbf{q})
\end{array}\right]
$$

with:

- $A_{m}\left(\mathbf{q}_{m}\right)$ the $n_{m} \times n_{m}$ inertia matrix of the manipulator;

- $\bar{A}_{p}(\mathbf{q})$ the $\left(n_{\text {dof }}-n_{m}\right) \times\left(n_{\text {dof }}-n_{m}\right)$ constraint consistent inertia matrix of the wheeled platform without accounting for the presence of the manipulator;

- $\bar{A}_{m p}(\mathbf{q})$ the $\left(n_{\text {dof }}-n_{m}\right) \times\left(n_{\text {dof }}-n_{m}\right)$ constraint consistent inertia matrix term representing the influence of the presence of the manipulator on the wheeled platform;

$-\bar{A}_{p / m}(\mathbf{q})$ the $n_{m} \times\left(n_{d o f}-n_{m}\right)$ constraint consistent inertia matrix representing the influence of the platform acceleration on the manipulator inertial properties.

$-\bar{C}(\mathbf{q}, \dot{\mathbf{q}})$ is the $n_{\text {dof }} \times n_{\text {dof }}$ constraint consistent matrix of the Coriolis and centrifugal effects of the system which can also be decomposed in terms of manipulator, platform and mutual effects; 
$-\overline{\boldsymbol{\Gamma}}=\bar{M} \boldsymbol{\Gamma}$, with $\bar{M}=S(\mathbf{q})^{t} M$

Given any (u, $\dot{\mathbf{u}}$ ) computed from (20) and any consistent $\mathbf{f}_{c}$ (consistent with $\dot{\boldsymbol{\xi}}$ in terms of controlled operational direction), one can compute the needed torque $\boldsymbol{\Gamma}_{o p}$ to fully compensate for the dynamic effects of the desired motion of the system:

$$
\overline{\mathbf{\Gamma}}_{o p}=\bar{A}(\mathbf{q}) \dot{\mathbf{u}}+\bar{C}(\mathbf{q}, \dot{\mathbf{q}}) \mathbf{u}+\overline{\mathbf{g}}\left(\mathbf{q}_{b}\right)+\bar{B}(\mathbf{q})^{t} \mathbf{f}_{c}
$$

and:

$$
\boldsymbol{\Gamma}_{o p}=\bar{M}^{\star}\left(\bar{A}(\mathbf{q}) \dot{\mathbf{u}}+\bar{C}(\mathbf{q}, \dot{\mathbf{q}}) \mathbf{u}+\overline{\mathbf{g}}\left(\mathbf{q}_{b}\right)+\bar{B}(\mathbf{q})^{t} \mathbf{f}_{c}\right)
$$

The obtained explicit formulation of the dynamic model allows to conclude that (28) leads to an actuation torque that perfectly compensate for the dynamic effects of the system providing that the weighting matrix for the weighted pseudo-inverse of matrix $\bar{B}(\mathbf{q})$ is chosen as the inertia matrix $\bar{A}$ of the system (see ${ }^{18}$ for details). In the case of force-based potential functions to locally optimise, access to the redundancy is also given in the torque space:

$$
\overline{\boldsymbol{\Gamma}}=\overline{\boldsymbol{\Gamma}}_{o p}+\left(\mathcal{I}_{n_{\text {dof }}}-\bar{B}(\mathbf{q})^{t} \bar{B}(\mathbf{q})^{t[1,2]}\right) \overline{\boldsymbol{\Gamma}}_{0}
$$

$\bar{B}(\mathbf{q})^{t[1,2]}$ (see ${ }^{19}$ for a complete definition of $[1,2]$-generalized inverses) also has to be chosen as the inertia matrix weighted pseudo-inverse in order to fully compensate for the system dynamic.

This framework can be extended to the operational space formulation of O. KнAтIB ${ }^{30}$ that was primarily developed for manipulators.

\section{Reactive control for wheeled mobile manipulators}

Using model-based actuation torque or velocity computation as presented in section 3, a framework has been developed allowing a reactive execution of complex mobile manipulation missions based on the sequencing of operational space tasks for the WMM. This was validated in simulation using a Matlab/Simulink simulator developed to simulate the dynamics of the WMM, test different control laws and implement our framework for reactive control. This framework was implemented using the $G^{e n}$ om control architecture ${ }^{31}$ of the LAAS Laboratory in Toulouse (France) on a real robot: $\mathrm{H}_{2} \mathrm{Bis}+\mathrm{GT6a}$.

\subsection{Framework description}

Missions based on the sequencing of operational tasks whose nature can be different are considered: free-space motion, constrained motion, force interaction. A mission can then be represented as a state machine where each state or mode represents a specific operational task. The possible transitions between states are described and transition conditions are mainly based on sensors information. This allows to describe a large number of missions. 
Regarding the operational trajectories, they can either be pre-planned or generated on-line. The actuators' inputs are then computed based either on inverse kinematics or on both inverse kinematics and dynamics. In both cases, the computed input can be written:

$$
\mathbf{c}=\underbrace{\mathbf{c}_{o p}}_{\text {operational task }}+\underbrace{\left(\mathcal{I}_{n_{\text {dof }}}-E(\mathbf{q})^{\star} E(\mathbf{q})\right) \mathbf{c}_{0}}_{\mathbf{c}_{\text {red }}: \text { redundancy }},
$$

where, in the kinematic case, $\mathbf{c}=\mathbf{u}, \mathbf{c}_{o p}=\bar{B}(\mathbf{q})^{\star} \dot{\mathbf{z}}, E(\mathbf{q})=\bar{B}(\mathbf{q})$ and $\mathbf{c}_{0}=\mathbf{u}_{0}$. In the torque formulation (dynamic or even static when neglecting inertial effects) : $\mathbf{c}=\overline{\boldsymbol{\Gamma}}, \mathbf{c}_{o p}=\overline{\boldsymbol{\Gamma}}_{o p}, E(\mathbf{q})=\bar{B}(\mathbf{q})^{t \star}$ and $\mathbf{c}_{0}=\overline{\boldsymbol{\Gamma}}_{0}$.

Actuator input Considering the case where redundancy is formulated in the kinematic framework, rated input actuators (in terms of velocity and acceleration) have to be respected. To do so, one has to ensure that the operational task term is such that:

$$
\left|\mathbf{c}_{o p}\right| \leq \mathbf{c}_{\max }
$$

where $\mathbf{c}_{\max }$ is the vector of maximum velocities for the actuators of the system. This constraint can be easily met when designing the input trajectories. One then has to find the largest positive value of a real $\alpha_{s}$ such that:

$$
\left|\mathbf{c}_{o p}+\alpha_{s} \mathbf{c}_{r e d}\right| \leq \mathbf{c}_{\max }
$$

This allows to scale down (or eventually up) the redundancy term in order to respect the constraint on maximum velocities. It also keeps the direction of descent of the gradient method used to compute $\mathbf{c}_{0}$, when it is chosen as a potential function to locally optimise. $\alpha_{s}$ can be computed as:

$$
\min \left(\left(\frac{\left|c_{\max , i} \cdot \operatorname{sign}\left(c_{r e d, i}\right)-c_{o p, i}\right|}{\left|c_{r e d, i}\right|}\right)_{i=1 \ldots n_{\text {dof }}}, \alpha_{s_{\max }}\right)
$$

This lead to a value of $\alpha_{s}$ between 0 and $\alpha_{s_{\max }} \cdot \alpha_{s_{\max }}$ is an upper limit used not to over amplify the redundancy term. It can for example be chosen using Armijo's rule.

Active constraint The criticalities of the constraints listed in Introduction can be different given the type of task to be executed. Moreover, those constraints are not always active. For example, the obstacle avoidance constraint has to be respected at any time. However, it is not be necessary to take it into account when far from obstacles. The same reasoning can be applied to any type of constraint and a deterministic arbitration mechanism was designed that evaluates the criticality at each time, with respect to the current executed task, of each potential function 
associated to the constraints and chooses which constraint is of greater importance at a given time. To avoid fast switching between two antagonist constraints, activation and deactivation thresholds for a given constraint can be chosen differently. A simple way to obtain smooth transition is to design a switching function $\beta_{s}(t)$ linearly varying between 0 and 1,0 being the value of the function when the transition starts (time $\left.t_{t i}\right)$ and 1 its value when the transition ends (time $\left.t_{t f}\right)$ :

$$
\beta_{s}(t)=\left\{\begin{array}{ccc}
0 & \text { if } & t<t_{t i} \\
\left(\frac{1}{\left(t_{t f}-t_{t i}\right)}\right)\left(t-t_{t i}\right) & \text { if } & t_{t i} \leq t<t_{t f} \\
1 & \text { if } & t \geq t_{t f}
\end{array}\right.
$$

The transition potential function is thus respectively given by :

$$
\mathcal{P}(\mathbf{q})=\left\{\begin{array}{c}
\mathcal{P}_{\text {old }}(\mathbf{q}) \\
\mathcal{P}_{\text {new }}(\mathbf{q}) \beta(t)^{2}+\mathcal{P}_{\text {old }}(\mathbf{q})\left(1-\beta(t)^{2}\right) \\
\mathcal{P}_{\text {new }}(\mathbf{q})
\end{array}\right.
$$

In order to ensure an efficient optimisation of the potential function associated to a given constraint, one has to make sure that the ratio $\frac{\mathbf{c}_{r e d}}{\mathbf{c}_{0}}$ is not too small and that $\alpha_{s}$ is not to small. The first case corresponds to a situation where the operational task to be achieved and the potential function to be optimised are not compatible. In that case, O. BRock et al. propose in ${ }^{8}$ to release some operational constraints temporarily in order to respect the constraints associated to the potential function. The second case occurs when the operational trajectories are very demanding in terms of actuation in which case the scaling of the redundancy term can lead to a drastic reduction of the efficiency of the optimised function. A threshold based mechanism is used (threshold on $\alpha_{s}$ value) leading to the modification of the operational trajectories in terms of speed of execution. This allows to redistribute the actuation capabilities of the system. In case of pre-planned trajectories, no complete re-planning is necessary and a simple adaptation of the original plan is sufficient. When trajectories are generated on-line, they are filtered using a low pass filter that is designed with varying parameters so that the constraints on the actuators inputs are met and so that the actuation power of the system can be redistributed. The direction of the operational movement is kept identical, only the speed of execution is reduced.

\subsection{Task sequencing involving contact transition}

The task sequencing mechanism was first introduced in ${ }^{32}$ in the case of a mission involving contact transition between free-space motion and constrained motion. In the example considered here, a free space motion is first planned for the end-effector followed by a constrained motion along a given surface. For the constrained motion, an hybrid force/velocity scheme is used where the normal direction to the contact surface is force-controlled and all the other operational directions are velocity-controlled. The WMM used for experimentation can only be velocity 
controlled and thus a kinematic-based controller is used.

In the case of contact transition, one more constraint is added to the list of constraints to be fulfilled : impact effects limitation at contact transition. Indeed, even if this transition is pre-planned, the uncertainties in the knowledge of the contact surface characteristics but also the uncertainties in the knowledge of the location of this contact surface can lead to a huge peak of force when contact occurs. This peak can be destructive for the environment and the WMM itself. One way to reduce this force peak is to design a potential function whose optimisation leads to a reconfiguration of the WMM such as it exhibits good inertial properties. Results concerning omnidirectional WMM are presented $i^{4}$. In this work the notion of impulse force $\hat{\mathcal{F}}$ is used but was first used in Robotics by I.D. WALKER in his study of contact transition for robotic arms ${ }^{33} . \hat{\mathcal{F}}$ is defined as:

$$
\hat{\mathcal{F}}=\lim _{\Delta t \rightarrow 0} \int_{t_{i}}^{t_{i}+\Delta t} \mathbf{f}(s) d s
$$

where $t_{i}$ is the impact time and $\mathbf{f}$ the force measured at the end-effector of the arm. Using the dynamic model of the system and basic collision theory, one can show that the magnitude of $\hat{\mathcal{F}}$ can be expressed as:

$$
\hat{F}=\frac{(1+e) \mathbf{v}^{t} \mathbf{n}}{\mathbf{n}^{T} R(\mathbf{q}) \mathbf{n}}
$$

$\mathbf{v}$ is the linear velocity of the end-effector at impact time, $e$ is the restitution coefficient (i.e. a physical characteristic of the contact), $\mathbf{n}$ is the normal direction to the contact surface and $R(\mathbf{q})$ is the inverse of the inertia matrix of the system projected in the operational space and restricted to linear acceleration terms. In order to minimize $\hat{F}$, one has of course to plan a low impact velocity but uncertainties in the knowledge of the environment location may still lead to bad results. $e$ can also be adapted using Remote Center Compliant Devices but thus leading to a very specific solution for the problem to be treated. In fact, the best solution is to maximize $\mathbf{n}^{T} R(\mathbf{q}) \mathbf{n}$ and thus to modify the configuration of the system in a way that will reduce the inertia of the system, seen at the end-effector, in the normal direction to the contact surface.

Both simulation and experimental results are presented. Those results illustrate the whole framework :

- operational tasks switching;

- reactive potential functions switching;

- contact transition;

- operational velocity adaptation.

In simulation, the WMM is a unicycle wheeled platform with a two dofs, revolute joints, planar arm. The position of the end-effector is controlled, not its orientation. The obstacles in the scene are not known at the planning 


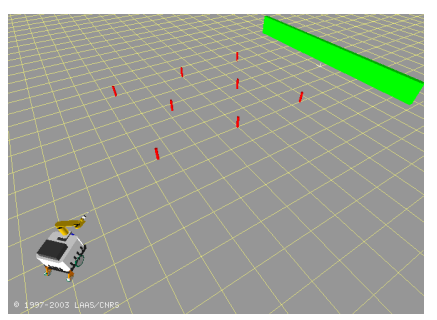

(a) Free space motion

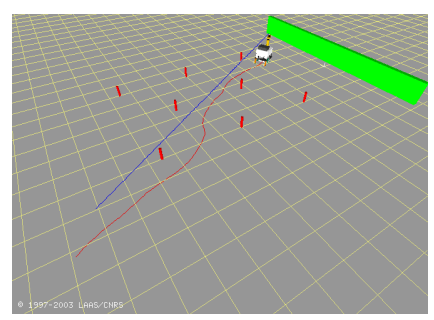

(b) Free space motion with contact transition active constraint

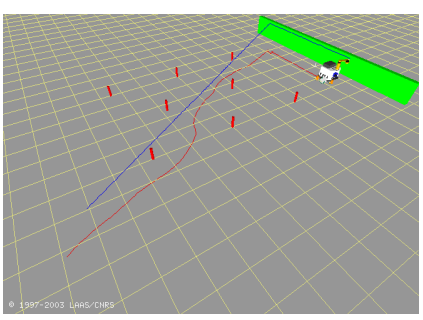

(c) Constrained motion

FIG. 3 - WMM mission with contact transition : decomposition in three tasks

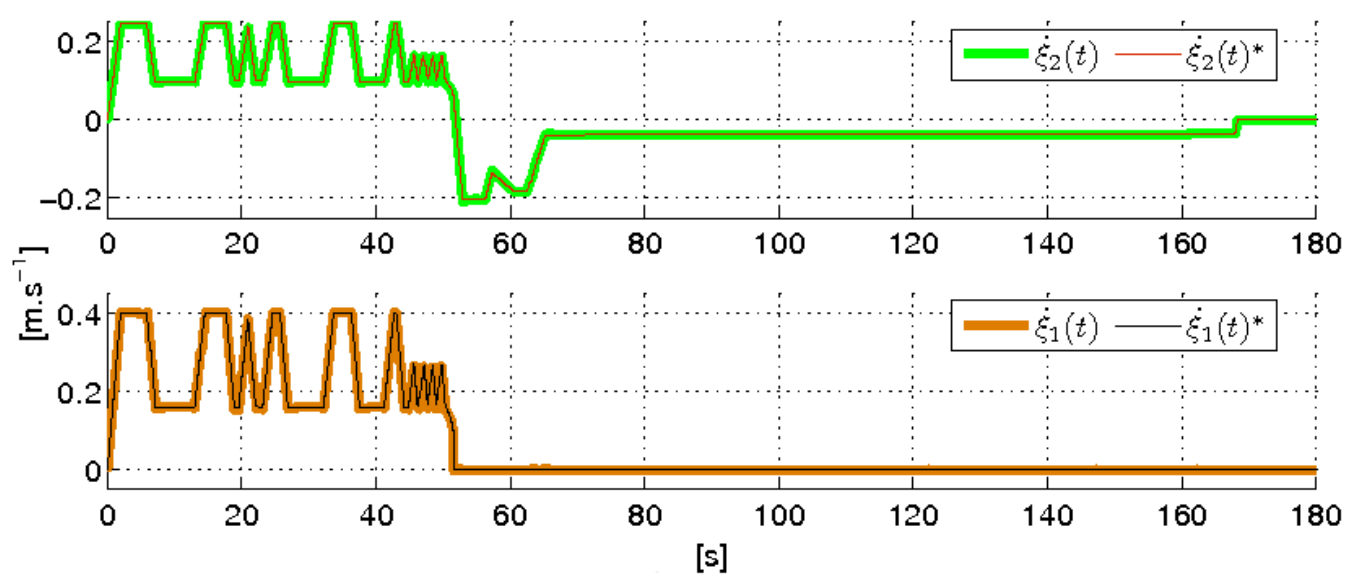

FIG. 4 - Adapted operational input velocities and real velocities

stage and thus have to be avoided dynamically using the redundancy of the system and an associated potential function. Figure 3 gives an overview of the different tasks associated to the mission. The operational trajectory is planned but its time execution is reactively modified to satisfy the different constraints. The planned velocity profile is trapezoidal from the initial point to the impact point. The latter is planned to be reached at zero velocity. Then, a second trapezoidal profile is planned for the end-effector velocity component tangential to the board. The resulting velocity profile is illustrated in figure 4. This figure presents the actual operational speed (reactively modified $\left(^{\star}\right)$ and real) of the end effector. The time execution of the end-effector motion is modified by this reactive adaptation but the end-effector path is not. The evolution of the value of the indicator associated to the active constraint is shown in figure 5. By comparing figure 4 and 5, one can notice that obstacle avoidance (see peaks in figure 5 corresponding to each new obstacle) is very demanding in terms of actuation and thus lead to the largest slowdowns of the operational velocity (figure 4). Regarding the constraint associated to manipulability, it is used to avoid singular or badly conditioned configuration for the system that would lead to a failure of the control input computation.

To illustrate the efficiency of the impact reduction strategy, results originally presented in ${ }^{34}$ are recalled. The normal 


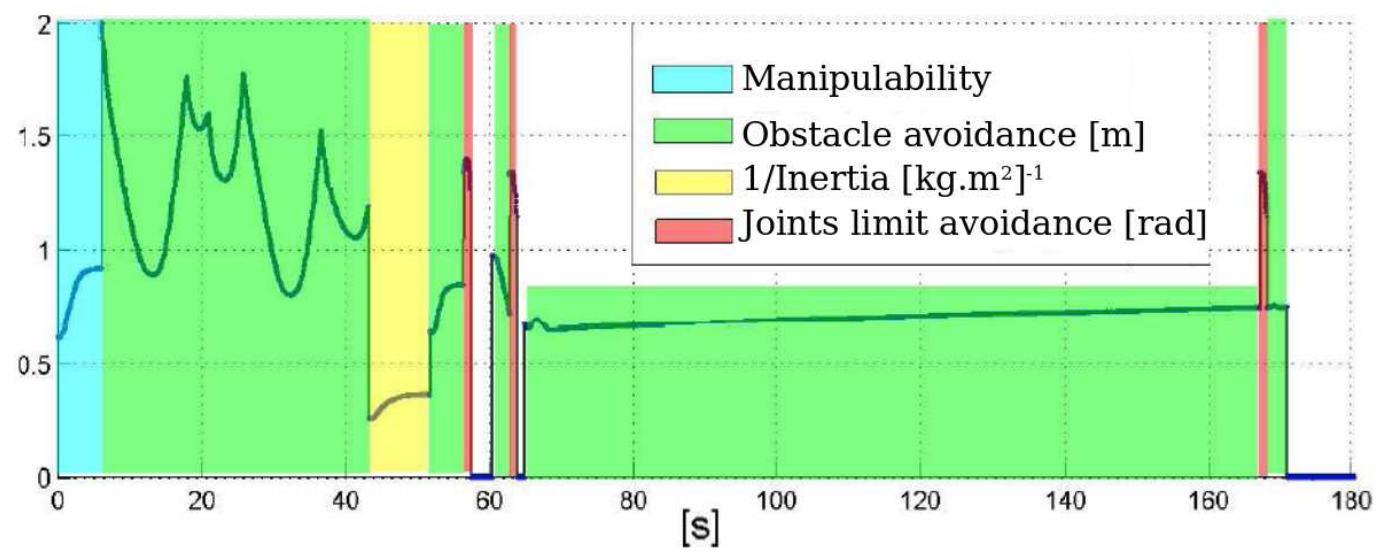

FIG. 5 - Evolution of the indicator associated to the active constraint

force set-point is $10 \mathrm{~N}$ and figure 6 shows that at contact time, the force overshoot is reduced from $80 \%$ to $20 \%$

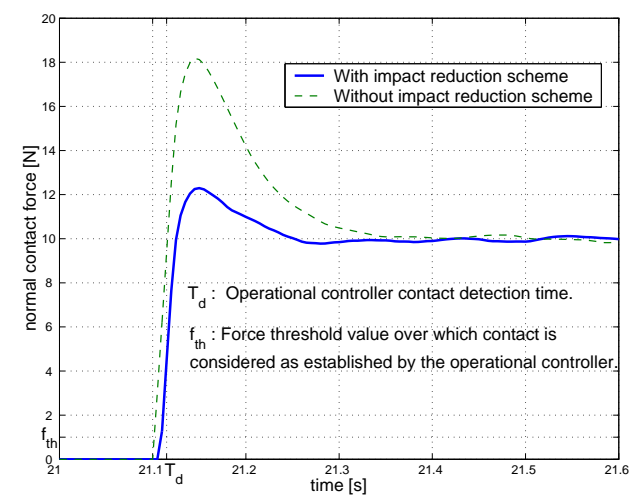

FIG. 6 - Impact force reduction : Normal contact force comparison.

when using the presented impact reduction strategy. Figure 7 illustrates the impact of this strategy on the velocities and configuration of the system. In the case of this specific WMM, the reduced inertia matrix is mostly a function of the arm configuration ( $q_{b 1}$ and $q_{b 2}$ in figure 7 ) and this leads to a larger influence of the impact scheme on the arm configuration than on the platform one $\left(\vartheta, x_{O_{p}}\right.$ and $y_{O_{p}}$ in figure 7$)$.

The experimental system is also composed of a unicycle wheeled platform and of a six dofs, revolute joints robotic arm. The whole system is controlled using on board calculators equipped with the real time operating system VxWorks and running on Motorola 68000 and Power PC boards. Control algorithms are implemented using $G^{e n}$ om, a generator of software control modules developed, as well as the robot, in the RIA team of the LAAS laboratory. At actuator level, the control sampling time is $5 \mathrm{~ms}$ (the smaller one admitted by the control modules) whereas at operational level it is chosen to be either 10 or $20 \mathrm{~ms}$.

The same type of contact transition mission has been tested using this experimental system. Figure 8 illustrates the initial and final configuration of the system. Figure 9 shows the linear velocity and position of the end-effector during the mission. The operational positioning error has a value of $5 \mathrm{~mm}$ at the end of the mission which is, in 


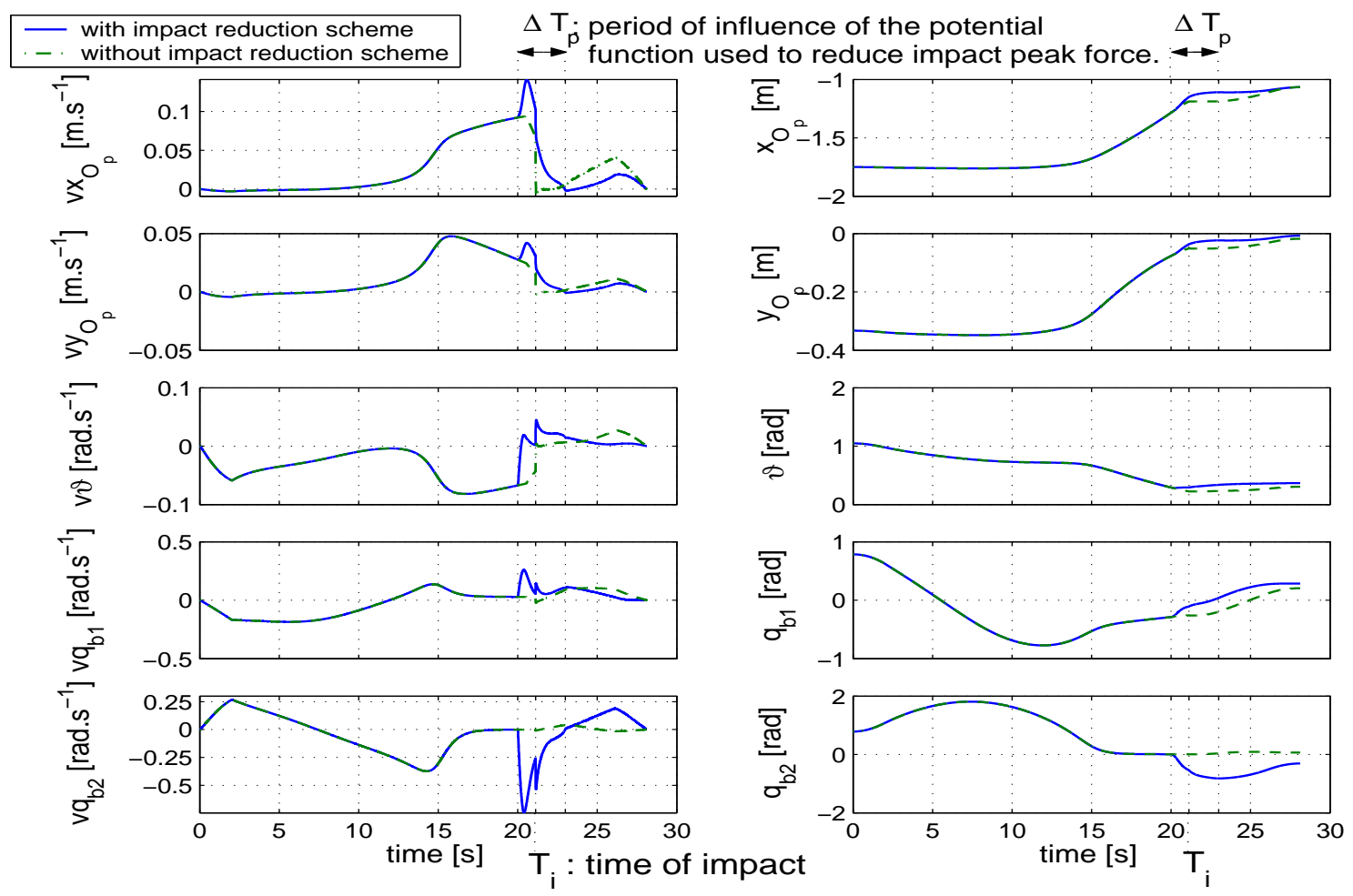

FIG. 7 - Impact force reduction : Effects on the position and speed of the platform and arm

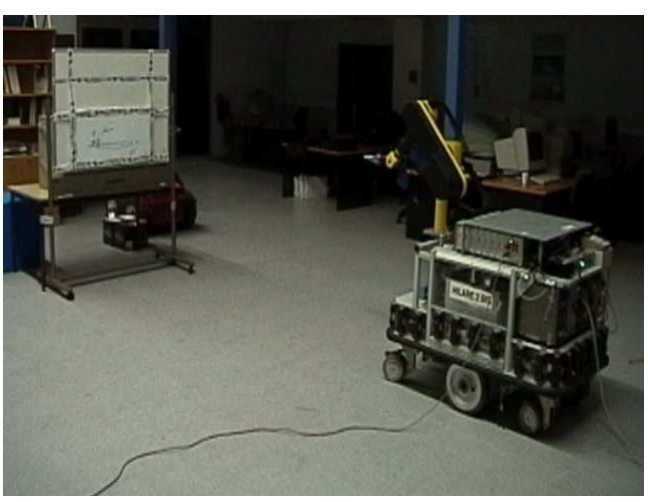

(a) System's initial configuration

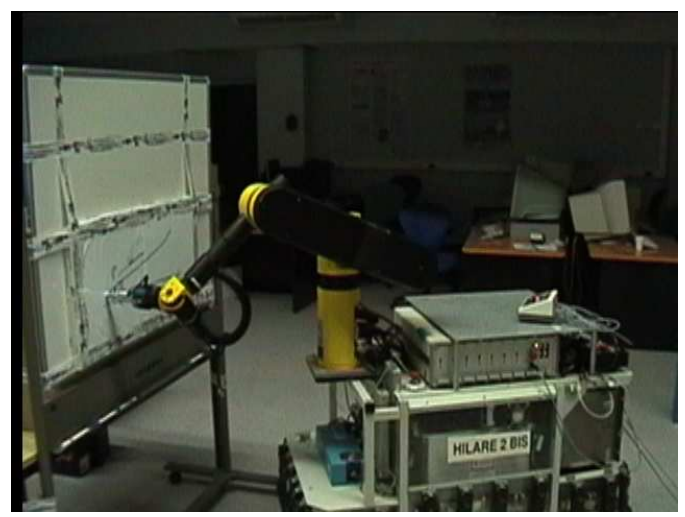

(b) System's final configuration

FIG. 8 - Initial and final configuration of the system for an experimental mission including contact transition.

the case of an indoor mobile manipulation mission, quite a good result in terms of tracking. The noise on the real velocity signals is actually not measurement noise but effects of high friction at the joint level when operating the robotic arm at slow velocity. Despite those uncompensated effects, the overall performance in terms of tracking (position and force) is good and demonstrate the effectiveness of the proposed approach. 

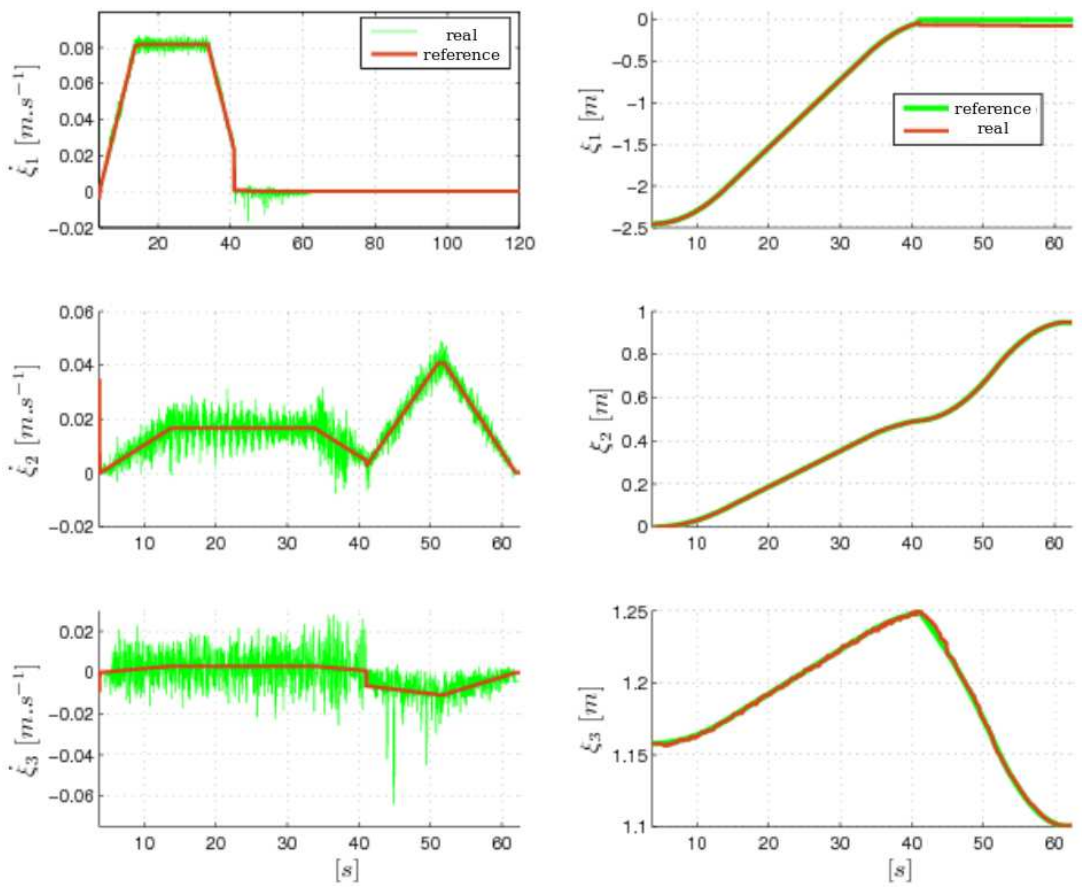

FIG. 9 - Linear velocity and speed of the end effector

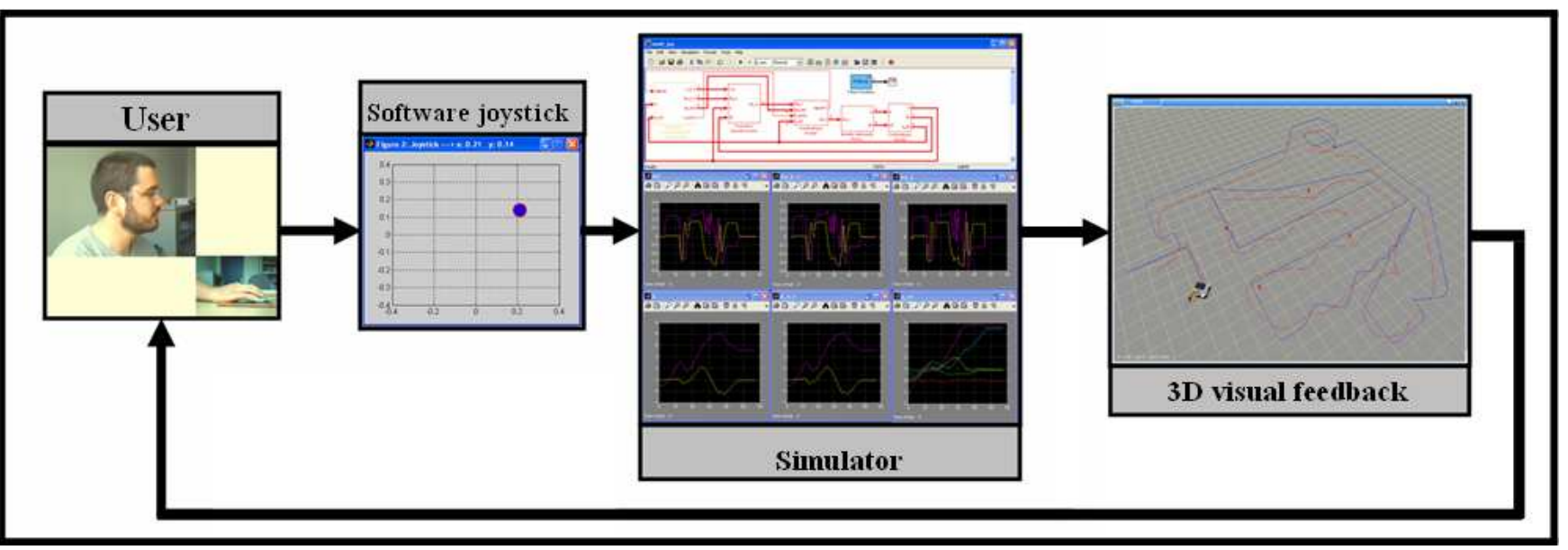

FIG. 10 - Principle of the virtual teleoperation of a wheeled mobile manipulator

\section{$4.3 \quad$ On-line generated operational trajectories}

To illustrate the case of on-line generated trajectories, simulation results are proposed, using the same planar WMM where the operational trajectory is generated in real time by a user interacting with Simulink via a software joystick. A 3D visual feedback of the scene is generated using $G D H E^{35}$. The principle of this application is given in figure 10.

For filtering purposes, a first order filter (low pass) $F(s)$ is chosen:

$$
F(s)=\frac{K_{f}}{1+\tau s},
$$




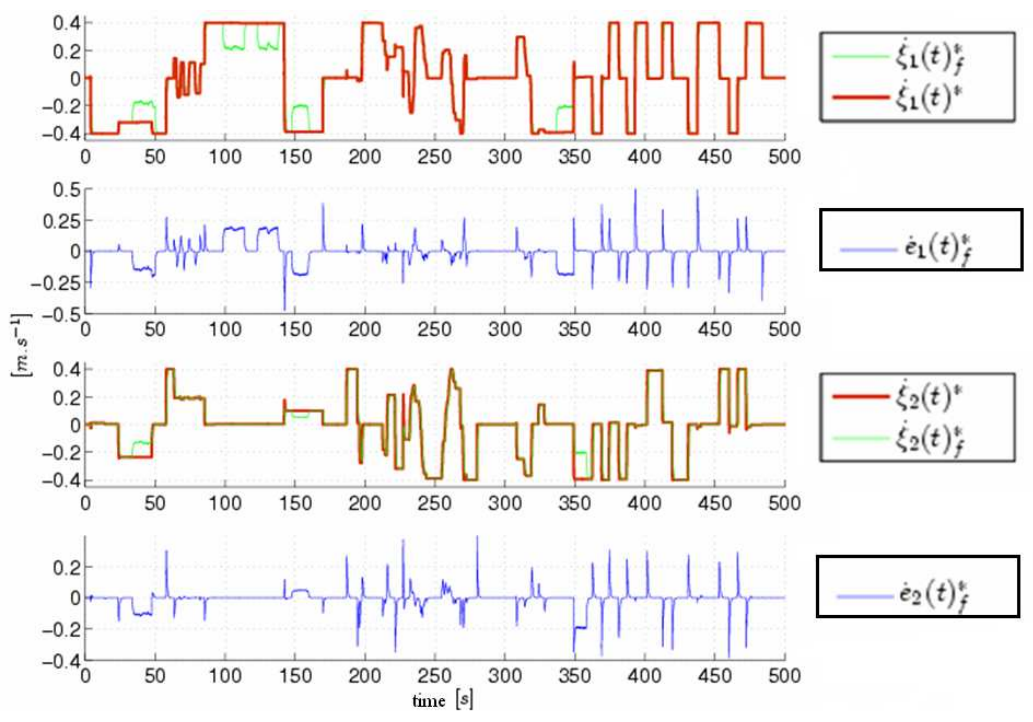

FIG. 11 - Effects of filtering operational velocity set-points issued from joystick

such as :

$$
\dot{\xi}(s)_{f}^{*}=F(s) \dot{\xi}(s)^{*},
$$

where $\dot{\xi}(t)^{*}$ is the norm of the operational velocity set-point generated by the user using the joystick and $\dot{\xi}(t)_{f}^{*}$ the norm of the filtered operational velocity set-point. Maximum operational velocity is dynamically set using $K_{f}$, a function of $\dot{\xi}_{\text {max }}, \mathcal{P}(\mathbf{q})$ and $\alpha_{s}$. Using relations (30) and (31) and choosing $\ddot{\xi}(t)_{f}^{*}$ to the maximum value $\ddot{\xi}_{\text {max }}$, parameter $\tau$ is given by :

$$
\tau=\frac{K_{f} \dot{\xi}(s)^{*}-\dot{\xi}(s)_{f}^{*}}{\ddot{\xi}_{\max }}
$$

For real time interaction purposes, simulation of the robot dynamics is simplified. Three different types of potential functions are considered:

- one for low obstacles avoidance; this potential function aims at maximising the distance from the wheeled mobile platform to the obstacles;

- one for each joint limit avoidance; these functions are bowl-shaped and take their maximum values around the joint limit values. They have to be minimized;

- one for numerical singularities avoidance; these singularities occur when $\bar{J}(\mathbf{q})$ is rank deficient or has a bad condition number; maximising the manipulability of the system is a way to avoid singular configurations.

Figure 11 presents the effect of filtering the operational velocity set-point issued from the joystick. The five time zones where the filtered velocity is really lower than the unfiltered one are the one for which obstacle avoidance is the 


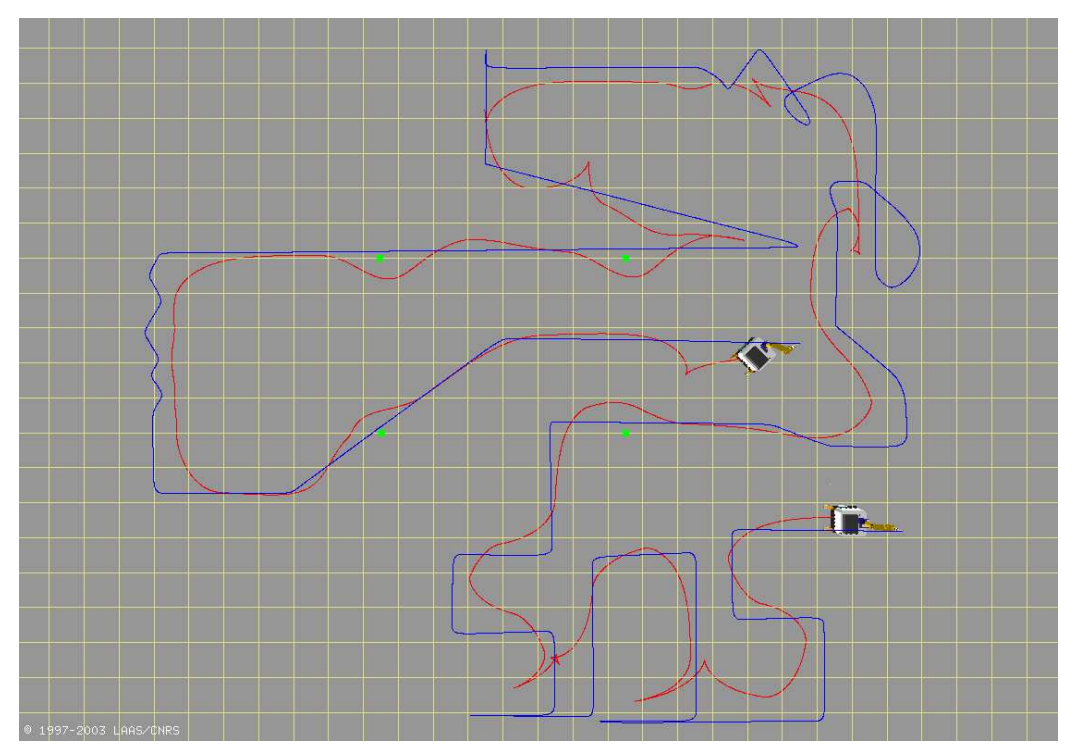

FIG. 12 - Planar view of the movement of the system during the mission

active constraint. In this particular simulation no joint limit avoidance was needed and when redundancy is not used to avoid low obstacles, it is used to maximize manipulability. This figure also presents signal $\mathbf{e}(t)_{f}^{*}=\dot{\boldsymbol{\xi}}(t)^{*}-\dot{\boldsymbol{\xi}}(t)_{f}^{*}$. This signal shows the effects of operational velocity set-point reduction as well as the difference induced by the respect of maximum operational acceleration.

Figure 12 gives a planar view of the whole movement (blue curve : path of the end-effector, red curve : path of point $O_{p}$ of the platform). Obstacles are represented as green points. These obstacles are efficiently avoided using a totally reactive control scheme. This robust reactive behaviour is also enlightened by the platform trajectory that exhibits not explicitly planned cusps.

\section{Conclusion}

In this paper, a unified framework for the reactive control of wheeled mobile manipulators is presented. It is dedicated to the execution of complex robotic missions composed of different types of operational tasks that are dynamically sequenced.

This framework is mainly based on model-based control strategies and relies on the generic kinematic and dynamic modeling of WMM. These models give access to the redundancy of the system. This redundancy allows, using local optimisation techniques, the satisfaction of the internal and external constraints associated to the control of such systems. Arbitration and switching between critical constraints, scaling of the actuators' inputs and operational trajectories offer the required flexibility in the realisation of a complex robotic mission. This framework has been implemented in both simulation and experimental cases. The exhibited performances demonstrate the feasibility and effectiveness of the proposed approach both in the case of pre-planned operational trajectories and on-line 
generated trajectories.

Further developments will be conducted especially to fully integrate and automate the design of the steering wheels' control within the proposed framework. In the case of outdoor applications, the rolling without slipping assumption may be too strong and it would be interesting to extend this framework to the case where a friction model of the couple wheel/ground might be useful in order to correctly model the dynamic behaviour of the WMM and thus correctly compensate for it.

\section{Web resources}

Movies of the simulation and experimental results can be found at:

http://www.stanford.edu/ vpadois/ResearcH/mobile_manipulation.html

\section{Acknowledgment}

This work was partially supported by the French CNRS RoBEA program within the projects:

- Advanced control techniques for wheeled mobile manipulators;

- Dynamic sequencing of multi-sensors based tasks for complex motions execution in mobile robotics.

\section{References}

1. H. Seraji, «An on-line approach to coordinated mobility and manipulation», in «Proceedings of the 1993 IEEE International Conference on Robotics and Automation», pp. 28-35 (Atlanta, USA, 1993).

2. Y. Yamamoto and X. Yun, Recent trends in mobile robots (Y. F. Zheng), chap. 6: Coordinating locomotion and manipulation of a mobile manipulator, pp. 157-181 (World Scientific, 1993).

3. Y. Yamamoto, «Control and coordination of locomotion and manipulation of a wheeled mobile manipulator», Ph.D. thesis, University of Pennsylvania, Philadelphia, USA (1994).

4. S. Kang, K. Komoriya, K. Yokoi, T. Koutoku and K. Tanie, «Utilization of inertial effect in damping-based posture control of mobile manipulator», in «Proceedings of the 2001 IEEE International Conference on Robotics and Automation», pp. 1277-1282 (Seoul, South Korea, 2001).

5. J. Tan and N. Xi, «Unified model approach for planning and control of mobile manipulators», in «Proceedings of the International Conference on Robotics and Automation», pp. 3145-3152 (Seoul, South Korea, 2001).

6. Y. Umeda, D. Nakamura, T. Mukarami and K. Ohnishi, «Hybrid Position/Force Control of a Mobile Manipulator based on Cooperative Task Sharing», in «Proceedings of the 1999 IEEE International Symposium on Industrial Electronics», (Bled, Slovenia, 1999). 
7. D. Omrčen, B. Nemec and L. Z̆lajpah, «Torque-velocity control algorithm for on-line obstacle avoidance for mobile manipulators», in «Proceedings of the 2003 International Conference on Industrial Technology», vol. 2, pp. 784-789 (Maribor, Slovenia, 2003).

8. O. Brock, O. Khatib and S. Viji, «Task consistent obstacle avoidance and motion behavior for mobile manipulation», in «Proceedings of the International Conference on Robotics and Automation», pp. 388-393 (Washington DC, USA, 2002), iSBN 0-7803-7273-5.

9. H. G. Tanner, S. Loizou and K. Kyriakopoulos, «Nonholonomic navigation and control of cooperating mobile manipulators», IEEE Transactions on Robotics and Automation, 19(1):53-64 (2003).

10. B. Bayle, M. Renaud and J.-Y. Fourquet, «Nonholonomic mobile manipulators: kinematics, velocities and redundancies», Journal of Intelligent and Robotic Systems, 36:45-63 (2003).

11. G. Campion, G. Bastin and B. D’Andréa Novel, «Structural properties and classification of kinematic and dynamic models of wheeled mobile robots», IEEE Transactions on Automatic Control, 12:47-62 (1996).

12. M. Fruchard, P. Morin and C. Samson, «A framework for the control of nonholonomic mobile manipulators», Rapport de recherche $\mathrm{N}^{\circ} 5556$ de l'Institut National de Recherche en Informatique et en Automatique, SophiaAntipolis, France (2005).

13. P. Morin and C. Samson, «Practical stabilization of driftless systems on Lie groups: the transverse function approach», IEEE Transactions on Automatic Control, 48:1496-1508 (2003).

14. B. Bayle, «Modélisation et commande cinématiques des manipulateurs mobiles à roues», Ph.D. thesis, Laboratoire d'Analyse et d'Architecture des Systèmes, Toulouse, France (2001).

15. D. T. Greenwood, Principles of Dynamics, second edition (Prentice Hall, 1988), iSBN 0-13-709981-9.

16. W. Khalil and J. Kleinfinger, «A new geometric notation for open and closed-loop robots», in «Proceedings of the 1986 International Conference on Robotics and Automation», vol. 2, pp. 1174-1179 (San Francisco, USA, 1986), iSBN 0-8186-0695-9.

17. B. Thuilot, B. d'Andrea-Novel and A. Micaelli, «Modeling and feedback control of mobile robots equipped with several steering wheels», IEEE Transactions on Robotics and Automation, 12(3):375-390 (1996).

18. V. Padois, «Enchaînements dynamiques de tâches pour des manipulateurs mobiles à roues», Ph.D. thesis, Institut National Polytechnique de Toulouse, France (2005).

19. A. Ben Israel and T. Greville, Generalized Inverses : Theory and Applications, second edition (Springer, 2003), iSBN 0-387-00293-6. 
20. F. G. Pin and J. C. Culioli, «Multi-criteria position and configuration optimization for redundant platform/manipulator systems», in «Proceedings of the 1990 IEEE International Workshop on Intelligent Robots and Systems», pp. 103-107 (1990).

21. Y. Nakamura, Advanced Robotics: redundancy and optimization (Addison Wesley, 1991), iSBN 0-201-15198-7.

22. P. Baerlocher and R. Boulic, «An inverse kinematic architecture enforcing an arbitrary number of strict priority levels», The Visual Computer, 20(6):402-417 (2004).

23. N. Mansard and F. Chaumette, «Directional redundancy: a new approach of the redundancy formalism», in «IEEE Conf. on Decision and Control and European Control Conference, CDC/ECC 2005», pp. 5366-5371 (Seville, Spain, 2005).

24. J. Chung and S. Velinsky, «Modeling and control of a mobile manipulator», Robotica, 16:607-613 (1998).

25. H. G. Tanner and K. Kyriakopoulos, «Mobile manipulator modeling with Kane's approach», Robotica, 19:675$690(2001)$.

26. Q. Yu and I.-M. Chen, «A general approach to the dynamics of nonholonomic mobile manipulator systems», Journal of Dynamic Systems, Measurement and Control, 124(4):512-521 (2002).

27. J. Nel̆mark and N. Fufaev, Dynamics of nonholonomic systems, vol. 33 of Translations of Mathematical Monographs (American Mathematical Society, 1972).

28. A. Bloch, J. Baillieul, P. Crouch and J. Marsden, Nonholonomic mechanics and control (Springer, 2003), iSBN $0-387-95535-6$.

29. J. Park and O. Khatib, «Multi-link multi-contact force control for manipulators», in «Proceedings of the 2005 IEEE International Conference on Robotics and Automation», pp. 3613-3618 (Barcelona, Spain, 2005).

30. O. Khatib, «A Unified Approach for Motion and Force Control of Robot Manipulators: The Operational Space Formulation», IEEE Journal of Robotics and Automation, 3(1):43-53 (1987).

31. S. Fleury, M. Herrb and R. Chatila, «GenoM: A tool for the specification and the implementation of operating modules in a distributed robot architecture», in «Proceedings of the Intelligent Robots and Systems», vol. 2, pp. 842-848 (Grenoble, France, 1997).

32. V. Padois, P. Chiron and J.-Y. Fourquet, «Controlling dynamic contact transition for nonholonomic mobile manipulators», in «Proceedings of the 2004 International Conference on Intelligent Robots and Systems», vol. 4, pp. 3817-3822 (Sendai, Japan, 2004), iSBN 0-7803-8464-4.

33. I. D. Walker, «Impact configurations and measures for kinematically redundant and multiple armed robot systems», IEEE Transactions on Robotics and Automation, 10(5):670-683 (1994). 
34. V. Padois, J.-Y. Fourquet, P. Chiron and M. Renaud, «On contact transition for nonholonomic mobile manipulators», in «Proceedings of the 9th International Symposium on Experimental Robotics», (Singapour, 2004).

35. M. Herrb, «GDHE - Graphical Display for Hilare Experiments : user guide», version 3.6 (2005).

\section{Appendix - List of symbols}

\section{Symbols in subsection 2.1}

$-n_{b}$ : Number of bodies of a multi-body system

$-p_{1}, p_{2}, \ldots, p_{6 n_{b}}:$ Primary generalized coordinates of the system

$-\sum_{i=1}^{6 n_{b}} \alpha_{i}\left(p_{1}, p_{2}, \ldots, p_{6 n_{b}}, t\right) \dot{p}_{i}+\beta\left(p_{1}, p_{2}, \ldots, p_{6 n_{b}}, t\right)=0$ : Generic form of a joint equation

$-f\left(p_{1}, p_{2}, \ldots, p_{6 n_{b}}, t\right)=0$ : Generic form of an holonomic joint equation

$-h_{p} \backslash h_{c} \backslash \bar{h}$ : Number of primary \complementary $\backslash$ nonholonomic joints equations

$-\mathbf{q} \backslash \dot{\mathbf{q}}$ : Vector of the $n$ generalized coordinates $\backslash$ velocities

$-n_{\text {dof }}=n-\left(h_{c}+\bar{h}\right):$ Global degree of freedom of the system

\section{Symbols in subsection 2.2}

$-\mathbf{q}_{m}$ : Vector of the $n_{m}$ generalized coordinates of the manipulator

$-\mathcal{R}=(O, \vec{x}, \vec{y}, \vec{z}):$ World reference frame

$-\mathcal{R}_{p}=\left(O_{p}, \vec{x}_{p}, \vec{y}_{p}, \vec{z}_{p}\right):$ Platform reference frame

$-\boldsymbol{\xi}_{p}=\left[\begin{array}{ccc}x & y & \vartheta\end{array}\right]^{t}$ : Vector of the $m_{p}$ location parameters of the platform in $\mathcal{R}$

- $N_{f}, N_{c}, N_{o c}, N_{s}$ : Number of fixed, centred, off-centred, Swedish wheels

$-N_{S}=N_{c}+N_{o c}:$ Number of steerable wheels

$-b, b^{\prime}, r, \alpha, \gamma, \beta, \varphi$ : Parameters describing the location of a wheel in $\mathcal{R}_{p}$

$-\boldsymbol{\varphi}=\left[\begin{array}{llll}\varphi_{f}^{t} & \varphi_{c}^{t} & \varphi_{o c}^{t} & \varphi_{s}^{t}\end{array}\right]^{t}$ : Spinning angles of the wheels

$-\boldsymbol{\beta}=\left[\begin{array}{ll}\boldsymbol{\beta}_{o c}^{t} & \boldsymbol{\beta}_{c}^{t}\end{array}\right]^{t}$ : Steering angles of the wheels

\section{Symbols in subsection $\mathbf{2 . 3}$}

$-C_{\alpha \beta} \backslash S_{\alpha \beta}$ : Shortcut notation of $\cos (\alpha+\beta) \backslash \sin (\alpha+\beta)$

$-R(\vartheta)$ : Rotation matrix of angle $\vartheta$ around $\vec{z}$

\section{Symbols in section 3}

$-\mathcal{R}_{E E}=\left(O_{E E}, \vec{x}_{E E}, \vec{y}_{E E}, \vec{z}_{E E}\right):$ End-effector reference frame 
$-\mathcal{R}_{m}=\left(O_{m}, \vec{x}_{m}, \vec{y}_{m}, \vec{z}_{m}\right):$ Manipulator reference frame

$-\boldsymbol{\xi} \backslash \boldsymbol{\xi}_{m}$ : Vector of the $m$ operational coordinates in $\mathcal{R} \backslash \mathcal{R}_{m}$

$-\boldsymbol{\xi}=\mathbf{f}\left(\boldsymbol{\xi}_{p}, \mathbf{q}_{m}\right)$ : Forward kinematics model of a WMM

- $\dot{\xi}$ : Operational velocities

- $J(\mathbf{q}): m \times n$ Jacobian matrix of a WMM

- $J_{1}, J_{2}, C_{1}, C_{2}$ : Rolling without slipping constraint matrices

- $D\left(\mathbf{q}_{p}\right)$ : Rolling without slipping compact constraint matrix

\section{Symbols in subsection 3.1}

$-C_{1 f c}\left(\boldsymbol{\beta}_{c}\right):$ Mobility constraint matrix

$-\delta_{\text {mobp }} \backslash \delta_{s t}$ : Degree of mobility $\backslash$ steerability of the platform

$-\mathcal{N}\left(C_{1 f c}\left(\boldsymbol{\beta}_{c}\right)\right):$ Kernel of $C_{1 f c}\left(\boldsymbol{\beta}_{c}\right)$

$-\Sigma\left(\boldsymbol{\beta}_{c}\right)$ : Motion compatibility matrix of the platform

$-\mathbf{u}_{m o b p} \backslash \mathbf{u}_{s t}$ : Mobility $\backslash$ steerability control input of the platform

$-\mathbf{u}_{\text {mobm }}$ : Mobility control input of the manipulator

- $\mathbf{u}$ : Vector of control inputs of a WMM

$-\delta_{m o b} \backslash \delta_{\text {man }}$ : Degree of mobility $\backslash$ manoeuvrability of a WMM

$-S\left(\mathbf{q}_{p}\right)$ : Motion compatibility matrix of a WMM

\section{Symbols in subsection 3.2}

- z: Extended operational coordinates vector of a WMM

- $B(\mathbf{q})$ : Extended jacobian of a WMM

$-\bar{J}(\mathbf{q})$ : Reduced jacobian of a WMM

- $\bar{B}(\mathbf{q})$ : Reduced extended jacobian of a WMM

- $\bar{B}(\mathbf{q})^{\star} \backslash \bar{J}(\mathbf{q})^{\star}$ : Weighted pseudo-inverse of $\bar{B}(\mathbf{q}) \backslash \bar{J}(\mathbf{q})$

$-\mathbf{u}_{0}$ : Vector of control inputs associated to the redundancy term

$-\mathcal{P}(\mathbf{q})$ : Configuration dependant potential function

$-\nabla \mathcal{P}(\mathbf{q}):$ Gradient of $\mathcal{P}(\mathbf{q})$

- $K$ : Positive definite weighting matrix

Symbols in subsection 3.3

$-T(\mathbf{q}, \dot{\mathbf{q}}, t)$ : Kinetic energy of a mechanical system

- P: Vector of the forces applied to the system due to its motion 
- Q: Vector of the sum of any other force acting on the system

- $\dot{\mathbf{q}}^{\star}$ : Virtual velocity of the system

$-\lambda$ : Vector of LAGRANGe multipliers

- $A(\mathbf{q}): n \times n$ symmetric, positive definite, inertia matrix of the system

- $C(\mathbf{q}, \dot{\mathbf{q}}): n \times n$ Coriolis and centrifugal matrix of the system

$-\Gamma$ : Vector of actuation forces provided by the $n_{a}$ actuators

- M: Matrix of actuation forces distribution in the system

$-\mathbf{Q}_{a}$ : Vector of actuation forces acting on the system

$-\mathbf{Q}_{g}=-\mathbf{g}(\mathbf{q})$ : Vector of gravity forces acting on the system

$-\mathbf{Q}_{d}$ : Vector of force disturbances such as friction effects acting on the system

- $\mathbf{Q}_{\text {ext }}$ : Vector of external forces acting on the system

$-\boldsymbol{\xi}_{c l}$ : Location on the system of the applied external forces

- $\mathbf{g}_{c l}(\mathbf{q})$ : Geometrical relation between the system configuration and $\boldsymbol{\xi}_{c l}$

$-J_{c l}(\mathbf{q})$ : Jacobian matrix associated to $\mathbf{g}_{c l}(\mathbf{q})$

$-J_{c}(\mathbf{q})$ : Matrix concatenation of the Jacobians for the $n_{c}$ external forces applied to the system

$-\mathbf{f}_{c}$ : Vector concatenation of the $n_{c}$ external forces applied to the system

$-\bar{A}(\mathbf{q}): n_{\text {dof }} \times n_{\text {dof }}$, symmetric, positive definite, constraint consistent inertia matrix of the system

$-A_{m}\left(\mathbf{q}_{m}\right): n_{m} \times n_{m}$ inertia matrix of the manipulator

$-\bar{A}_{p}(\mathbf{q}):\left(n_{\text {dof }}-n_{m}\right) \times\left(n_{\text {dof }}-n_{m}\right)$ constraint consistent inertia matrix of the wheeled platform without accounting for the presence of the manipulator

$-\bar{A}_{m p}(\mathbf{q}):\left(n_{\text {dof }}-n_{m}\right) \times\left(n_{\text {dof }}-n_{m}\right)$ constraint consistent inertia matrix term representing the influence the manipulator on the wheeled platform

$-\bar{A}_{p / m}(\mathbf{q}): n_{m} \times\left(n_{d o f}-n_{m}\right)$ constraint consistent inertia matrix representing the influence of the platform acceleration on the manipulator inertia

$-\bar{C}(\mathbf{q}, \dot{\mathbf{q}}): n_{\text {dof }} \times n_{\text {dof }}$ constraint consistent matrix of the Coriolis and centrifugal effects on the system

$-\overline{\boldsymbol{\Gamma}}$ : Constraint consistent vector of actuation forces

$-\boldsymbol{\Gamma}_{o p}$ : Computed actuation forces for a given operational motion $\backslash$ force and full dynamic compensation

$-\overline{\boldsymbol{\Gamma}}_{o p} \backslash \overline{\boldsymbol{\Gamma}}_{0}$ : Constraint consistent vector of actuation forces associated to the operational points $\backslash$ redundancy of the system

$-\bar{B}(\mathbf{q})^{t[1,2]}:$ A $[1,2]$-generalized inverse of $\bar{B}(\mathbf{q})^{t}$

\section{Symbols in subsection 4.1}

- c: Computed control input (either velocity or force $\backslash$ torque)

- $\mathbf{c}_{o p} \backslash \mathbf{c}_{0}, \mathbf{c}_{r e d}$ : Computed control input related to the operational points $\backslash$ redundancy of the system

$-\mathbf{c}_{\max }$ : Vector of maximum values for the control inputs

$-\alpha_{s}$ : Scaling factor for the redundancy term

$-\alpha_{s_{\max }}:$ Maximum value of $\alpha_{s}$

$-\beta_{s}(t)$ : Transition function between two secondary constraints 
$-t_{t i} \backslash t_{t f}:$ Starting $\backslash$ final time of the transition

- $\mathcal{P}_{\text {old }}(\mathbf{q})$ : Potential function associated to the secondary constraints before switching

$-\mathcal{P}_{\text {new }}(\mathbf{q})$ : Potential function associated to the secondary constraints after switching

\section{Symbols in subsection 4.2}

$-\hat{\mathcal{F}}$ : Impulse force at impact for contact transition

$-t_{i}$ : Time of impact for contact transition

$-\Delta t$ : Infinitesimal period of time during which impact occurs

- f: Measured force at the end-effector of the arm

$-\hat{F}$ : Estimated magnitude of $\hat{\mathcal{F}}$

- v : Linear velocity of the end-effector at impact time

$-e$ : Restitution coefficient of the contact

- n : Normal direction to the contact surface

$-R(\mathbf{q})$ : Inverse of the inertia matrix of the system projected in the operational space and restricted to linear acceleration terms

$-T_{d}$ : Operational controller contact detection time

$-f_{t h}$ : Force threshold value used for contact detection

$-\Delta T_{p}$ : Period of influence of the impact reduction scheme

\section{Symbols in subsection 4.3}

- $F(s)$ : Transfer function of the low pass filter used to adapt the norm of the end-effector desired velocity $\dot{\xi}(t)^{*}$

$-\tau \backslash K_{f}$ : Time constant $\backslash$ static gain of $F(s)$

$-\dot{\xi}(t)_{f}^{*} \backslash \ddot{\xi}(t)_{f}^{*}$ : Filtered value of the norm of the end-effector desired velocity $\backslash$ acceleration

$-\dot{\xi}_{\text {max }} \backslash \ddot{\xi}_{\text {max }}$ : Maximum value of the norm of the end-effector velocity $\backslash$ acceleration

- $\mathbf{e}(t)_{f}^{*}$ : Difference between the desired end-effector velocity vector $\dot{\boldsymbol{\xi}}(t)^{*}$ and the filtered one $\dot{\boldsymbol{\xi}}(t)_{f}^{*}$ 